\title{
RESPUESTA SÍSMICA DE TANQUES CILÍNDRICOS PARA ALMACENAMIENTO DE LÍQUIDOS
}

\author{
Hugo Hernández Barrios ${ }^{l}$ y Ernesto Heredia Zavoni ${ }^{1,2}$
}

\begin{abstract}
RESUMEN
Se estudia la respuesta sísmica de tanques cilíndricos para almacenamiento de líquidos. Se consideran tanques de pared rígida, anclados a la cimentación y que contienen fluidos ideales, irrotacionales e incompresibles. La ecuación de movimiento se plantea en coordenadas cilíndricas y se encuentra una solución analítica para el potencial de velocidad. El comportamiento sísmico se evalúa en términos de altura de ola máxima, fuerzas cortantes, momentos de volteo y presiones hidrodinámicas. El cálculo numérico de la respuesta se realiza paso a paso utilizando registros del sismo del 19 de septiembre de 1985 en el Valle de México y Caleta de Campos. Los resultados muestran la contribución de los modos superiores del oleaje en la respuesta sísmica y los efectos de resonancia en excitaciones de frecuencia dominante cercana a la frecuencia del modo fundamental del oleaje en la superficie libre del líquido. Se analizan los efectos de las componentes horizontales y vertical de la excitación en la respuesta.
\end{abstract}

\begin{abstract}
The paper studies the seismic response of cylindrical tanks for liquid storage. Tanks are assumed to have a rigid wall, to be anchored to a rigid foundation and to store an ideal and incompressible fluid. The problem is solved in cylindrical coordinates. The response is assessed in terms of maximum wave heights, shear forces, bending moments and hydrodynamic pressures. Numerical computations of the response are performed in the time domain using records from Mexico City and Caleta de Campos. The results show the contribution of higher wave modes and of resonance effects on the seismic response. The influence of the horizontal orthogonal components of ground motion, as well as that of the vertical component, on the response are analyzed.
\end{abstract}

Artículo recibido el 4 de julio de 2000 y aprobado para su publicación el 13 de septiembre de 2000. Se aceptarán comentarios y/o discusiones al artículo hasta cinco meses después de su publicación.

1 Instituto Mexicano del Petróleo Competencia de Ingeniería Civil Apdo. Postal 14-805, México DF 07730

hbarrios@www.imp.mx eheredia@www.imp.mx

2 Instituto de Ingeniería, UNAM

Mecánica Aplicada

Apdo. Postal 70-472, México DF 04510

eheredia@leviatan.iingen.unam.mx 


\section{INTRODUCCION}

Daños severos han ocurrido en tanques cilíndricos para almacenamiento de líquidos durante temblores como los de Alaska, 1964, Nigata, 1964, California, 1980, Colinga, 1983 y Northridge, 1994. Se han observado daños por pandeo en las paredes del tanque (ver Figura 1.a), daños en la cubierta por la magnitud del oleaje en la superficie del líquido (ver Figura 1b), daños en soldaduras y rupturas entre la pared del tanque y el anillo base, así como levantamientos del anillo de cimentación (Niwa y Clough, 1982; Manos y Clough, 1985; Sakar y Ogawa, 1984; Jennings, 1971). Recientemente, el sismo de Kocaeli en Turquía, 1999, produjo fallas en las conexiones de las tuberías de alimentación debidas a momentos de volteo excesivos.

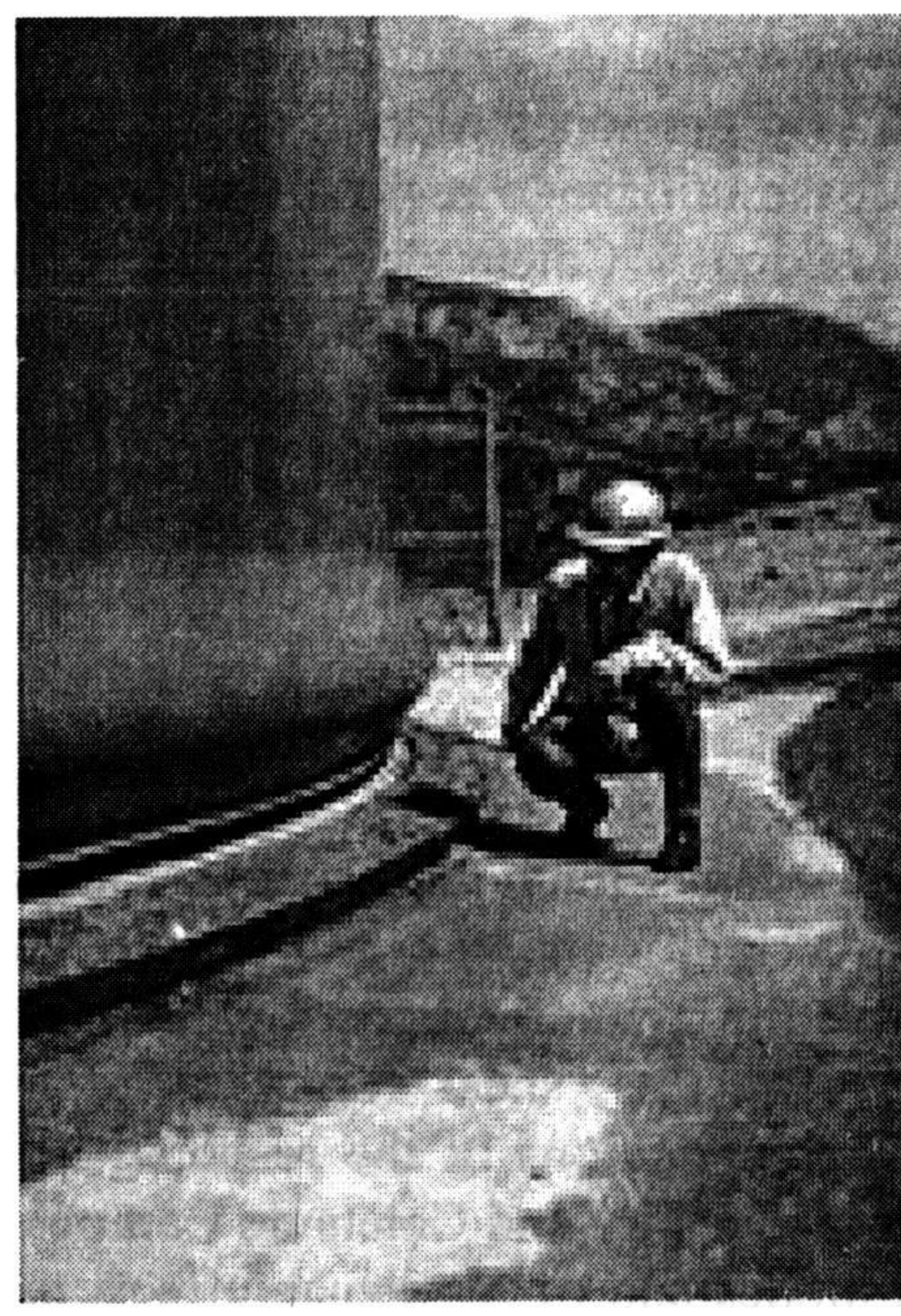

a) Falla por pandeo tipo "pata de elefante"

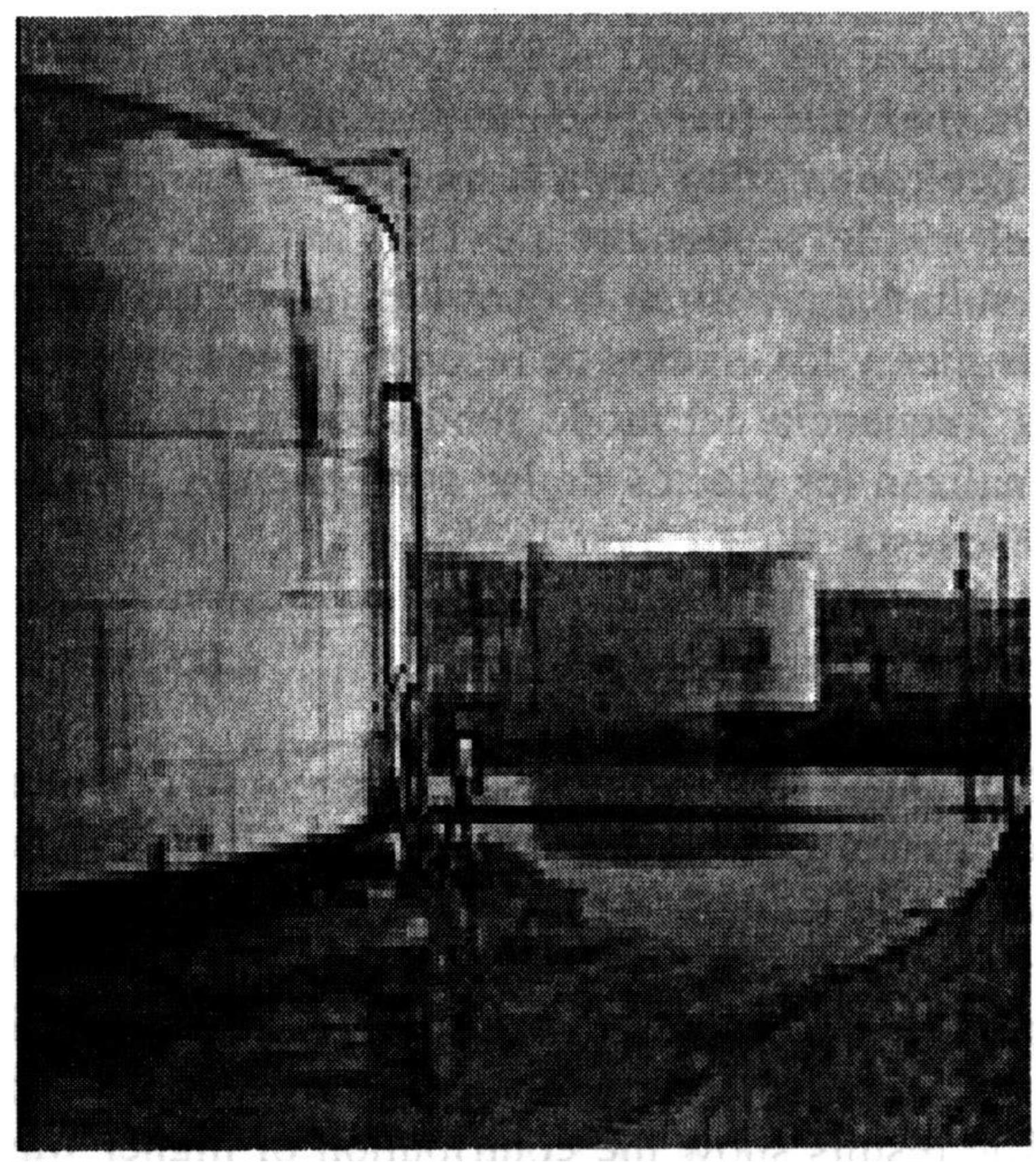

b) Falla en la cubierta debida al oleaje

Fig 1. Fallas en tanques cilíndricos debidas a sismos

En el Distrito Federal (OF), México, existen cerca de 250 tanques de almacenamiento y regulación de agua potable. La mayoría se ubica en las zonas de lomas y de transición, y más de una docena de tanques se encuentran en la zona de lago (ver Figura 2). En el área metropolitana se encuentran también un gran número de plantas de tratamiento de aguas negras y plantas industriales que cuentan con tanques cilíndricos de almacenamiento. Estos tanques son vitales para el sistema de abastecimiento y dosificación de agua potable.

Existen también en el área metropolitana del DF tanques de abastecimiento de la industria petrolera, asícomo tanques para almacenamiento de turbosina y gasavión. La falla de alguna d estas estructuras durante un temblor afectaría el abastecimiento de combustible 
para tráfico aéreo, causaría un gran daño ecológico y pondría en riesgo la vida de un gran número de personas.

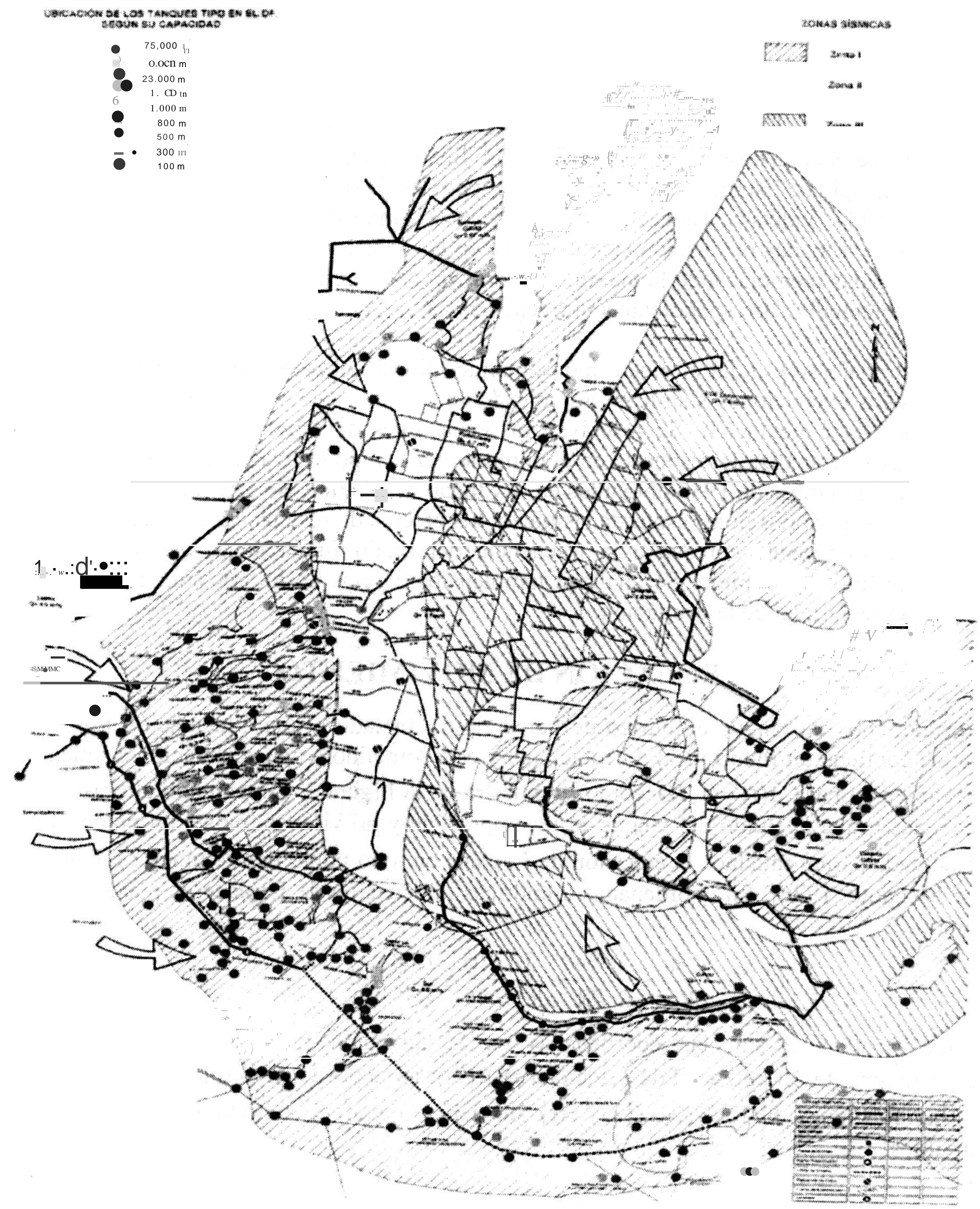

Fig 2. Ubicación de tanques de almacenamiento en el Distrito Federal 
Los códigos de diseño sísmico consideran métodos para el análisis sísmico de tanques. Los códigos API-STD-650-93, AWWA D1 00-88 y NZNSEE-1986, proponen un procedimiento de análisis basado en el modelo simplificado masa-resorte desarrollado por Housner (1957) y modificado por Veletsos (Veletsos y Yang, 1977). Dicho procedimiento considera dos modos de la respuesta del sistema fluido-tanque: (1) el del sistema cascarón-cubierta, junto con una porción del líquido que se mueve al unísono con éste, asociado a una fuerza impulsiva; y (2) el fundamental del oleaje del líquido contenido en el tanque asociado a una fuerza convectiva. Las hipótesis básicas en el análisis son: (1) la amplitud del oleaje es pequeña y se pueden despreciar los efectos hidrodinámicos no lineales; y (2) las paredes del tanque son rígidas. Estudios sobre tanques con paredes flexibles (Veletsos y Kumar, 1984; Veletsos, 1984; Veletsos, Tang Y. y Tang H. T., 1992) n1uestran que las fuerzas impulsivas pueden ser estimadas razonablemente con base en las soluciones obtenidas para tanques de paredes rígidas reemplazando la máxima aceleración del terreno por el valor espectral de la pseudoaceleración correspondiente a la frecuencia natural del fluido.

En este trabajo se estudia el efecto hidrodinámico lineal del oleaje en tanques cilíndricos sometidos a excitaciones sísmicas. Se consideran tanques anchos, con paredes rígidas y anclados a la cimentación. Se resuelven las ecuaciones de movimiento en coordenadas cilíndricas y se analiza la respuesta en el dominio del tiempo utilizando registros del sismo del 19 de septiembre de 1985 obtenidos en la Ciudad de México. Se estudia la influencia de los modos superiores en la respuesta sísmica y los efectos de resonancia en excitaciones de frecuencia dominante cercana a la frecuencia del modo fundamental del oleaje en la superficie libre del líquido. Se analizan también los efectos de bidireccionalidad y de la componente vertical de la excitación.

\section{ECUACIÓN DE MOVIMIENTO}

Consideremos un tanque cilíndrico de pared rígida anclado a una cimentación rígida. El tanque contiene un fluido ideal e irrotacional y está sometido a una aceleración sísmica del terreno en su base $\mathrm{a}(\mathrm{t})=\mathrm{ax}(\mathrm{t})$ como se muestra en la Figura 3. Suponiendo alturas de ola pequeñas y que el fluido del tanque es incompresible y no viscoso, existe un potencial de velocidad, cp, que satisface la ecuación de Laplace en todo punto del volumen $\mathrm{Q}, \mathrm{V}^{2} \mathrm{q}=\mathrm{O}$, (Le Méhauté, 1976). En coordenadas cilíndricas la ecuación de Laplace se puede escribir como:

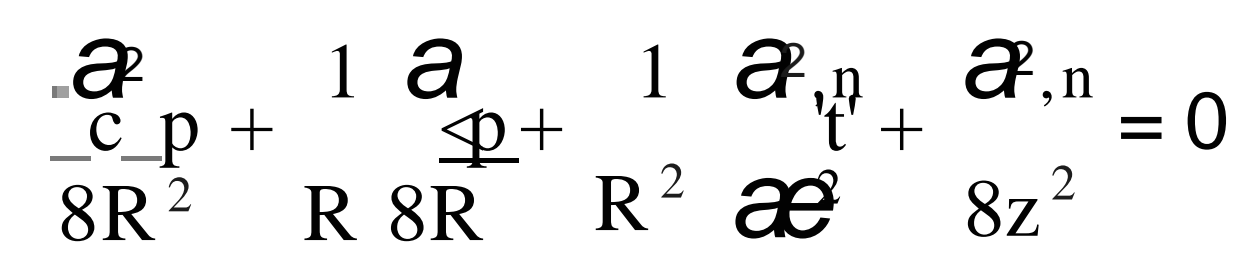

donde $\mathrm{R}, 8 \mathrm{y} \mathrm{z}$ son las coordenadas en la dirección radial, angular y vertical, respectivamente. El potencial de velocidad $\mathrm{cp}(\mathrm{R}, 8, \mathrm{z}, \mathrm{t})$ se puede expresar como el producto de las funciones 91(R), $\mathrm{T}(8), \mathrm{Z}(\mathrm{z})$ y $\mathrm{F}(\mathrm{t})$ de acuerdo con el método de separación de variables,

$$
\operatorname{cp}(\mathrm{R}, 8, \mathrm{z}, \mathrm{t})=\mathrm{m}(\mathrm{R}) \mathrm{T}(8) \mathrm{Z}(\mathrm{z}) \mathrm{F}(\mathrm{t})
$$




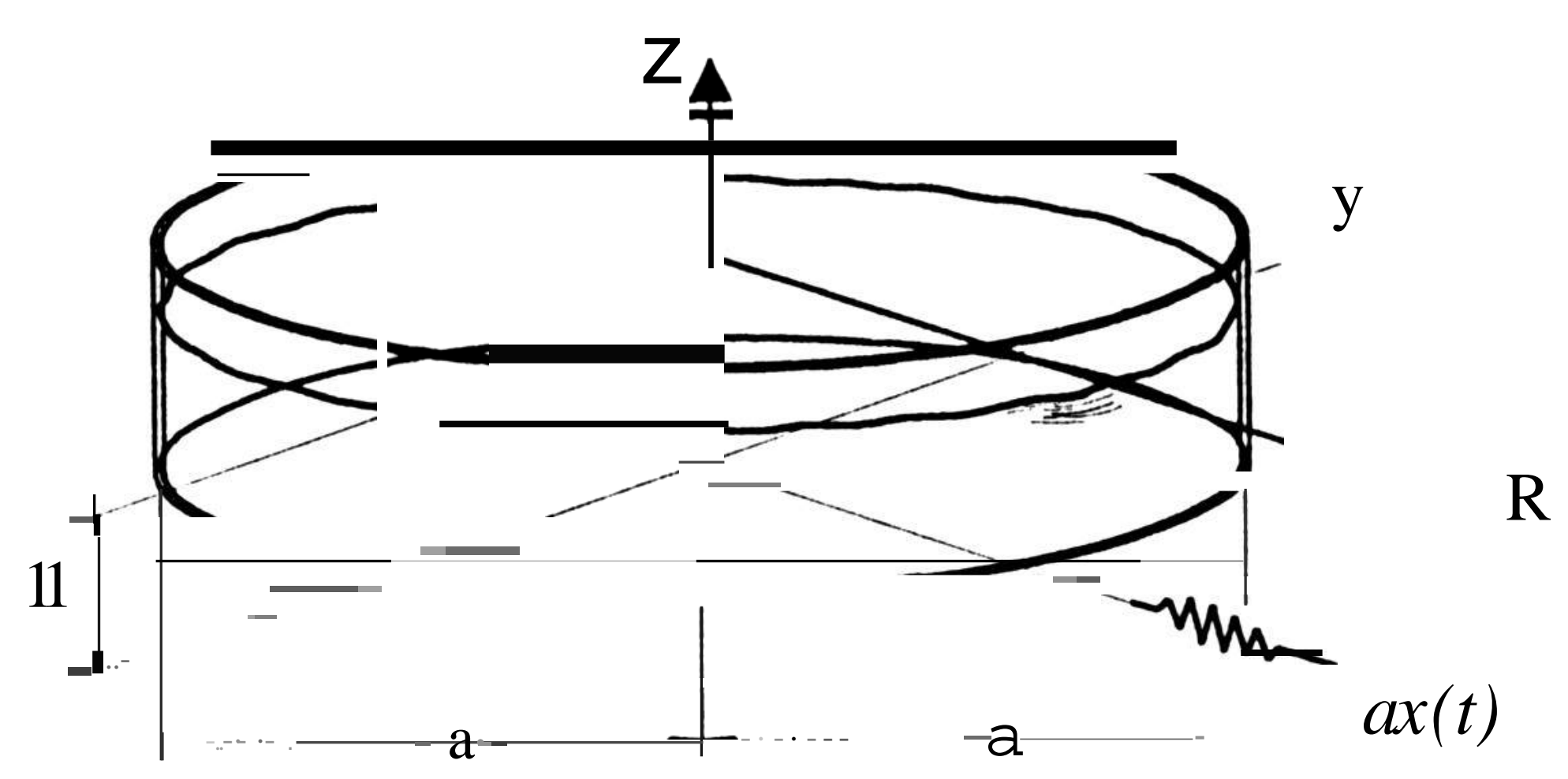

(a) Notación General

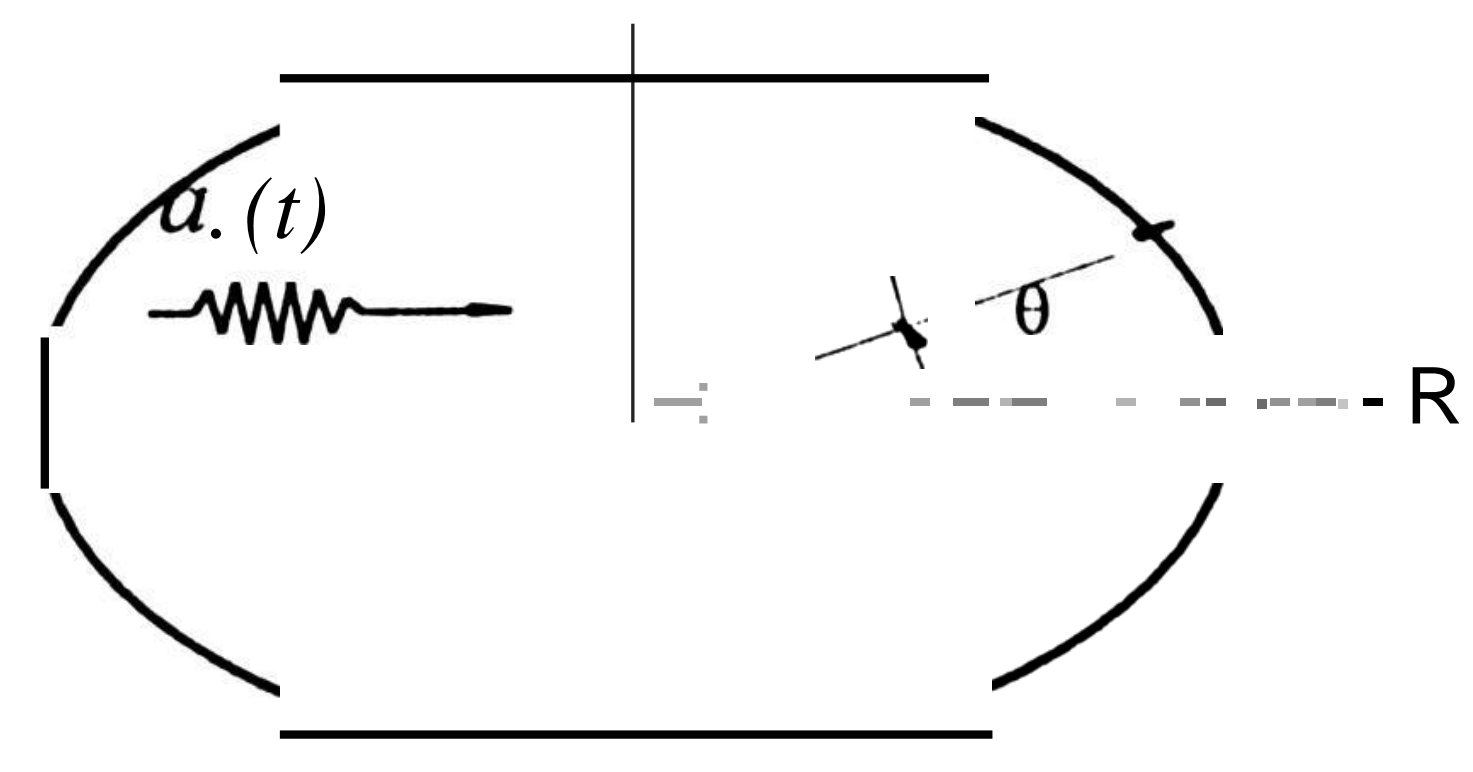

(b) Planta

Fig 3. Tanque cilíndrico en estudio

Sustituyendo (2) en (1) se obtienen las siguientes ecuaciones diferenciales ordinarias

$$
\begin{array}{r}
\mathrm{T}(8)+\mathrm{m}^{2} \mathrm{~T}(8)=\mathrm{O} \\
\mathrm{Z}(\mathrm{Z})-\mathrm{k}^{2} \mathrm{Z}(\mathrm{Z})=\ddot{\mathrm{U}} \\
\mathrm{R}^{2} \mathrm{gl}(\mathrm{R})+\mathrm{k}^{2} \mathrm{R}^{2}-\mathrm{m}^{2} \quad(\mathrm{R})=\mathrm{O}
\end{array}
$$

qonde $\mathrm{m}$ y $\mathrm{k}$ son constantes. La ecuación (3) es similar a la de vibración libre no amortiguada de un oscilador simple cuya solución es

$$
\mathrm{T}(8)=\mathrm{A}_{1} \operatorname{sen}(\mathrm{m} 8)+\mathrm{A}_{2} \cos (\mathrm{m} 8)
$$

La solución de la ecuación (4) se puede escribir como

$$
\mathrm{Z}(\mathrm{z})=\mathrm{B}_{1} \exp (\mathrm{kz})+\mathrm{B}_{2} \exp (-\mathrm{kz})
$$

La ecuación (5) es la forma canónica de la ecuación de Bessel de orden m y su solución es, 


$$
\mathfrak{R}(\mathrm{R})=\mathrm{C}_{\mathrm{m}} \mathrm{J}_{\mathrm{m}}(\mathrm{kR})+\mathrm{D}_{\mathrm{m}} \mathrm{Y}_{\mathrm{m}}(\mathrm{kR})
$$

donde $\mathrm{Jn}_{1}$ y $\mathrm{Ym}$ son funciones de. Bessel de primer y segundo tipo, respectivamente, de orden $\mathrm{m}$. Dado que $\mathrm{Ym}(\mathrm{kR})$ es singular en $\mathrm{R}=\mathrm{O}$ para todo valor de $\mathrm{m}$, los coeficientes de integración Dmdeben de ser cero, i.e. el potencial de velocidad es proporcional a Jm(kR).

$$
\mathfrak{R}(\mathrm{R})=\mathrm{C}_{\mathrm{m}} \mathrm{J}_{\mathrm{m}}(\mathrm{kR})
$$

Substituyendo (6),(7) y (9) en (2), el potencial de velocidad está dado por

$$
\begin{aligned}
\operatorname{cp}(\mathrm{R}, 8, \mathrm{z}, \mathrm{t})= & {\left[\mathrm{A}_{1} \operatorname{sen}(\mathrm{m} 8)+\mathrm{A}_{2} \cos (\mathrm{m} 8)\right]\left[\mathrm{B}_{1} \exp (\mathrm{zk})+\mathrm{B}_{2} \exp (-\mathrm{zk})\right] } \\
& {\left[\mathrm{C}_{\mathrm{m}} \mathrm{J}_{\mathrm{m}}(\mathrm{kR})\right] \mathrm{F}(\mathrm{t}) }
\end{aligned}
$$

donde las constantes de integración A1, A2, B1, B2 y Cm deben determinarse según las condiciones de frontera.

\section{CONDICIONES DE FRONTERA}

Consideremos un potenci al de velocidad referido a un sistema móvil cuyas coordenadas están referidas a un sistema inercial fijo, es decir, un potencial de velocidad absoluto con respecto a un marco relativo. Se puede demostrar que las condiciones de frontera referidas a un marco relativo son:

$$
\begin{aligned}
& \frac{2 p}{\delta R}=0 \text { en } R= \pm a \\
& \begin{array}{l}
a p-0 \\
C R
\end{array} \text { en } \mathrm{z}==-\mathrm{h}
\end{aligned}
$$

donde a es el radio del tanque $\mathrm{y} h$ la profundidad. La ecuación (11) indica que la componente del vector de velocidad normal a la pared del tanque es cero, es decir, la velocidad relativa entre el líquido contenido en el tanque y la pared del tanque es nula. La interpretación física de la ecuación (12) es similar a la anterior sólo que en el fondo del tanque. Ambas condiciones de frontera especifican que el líquido no traspasará las fronteras rígidas del tanque durante u movimiento.

En la superficie libre del líquido la condición cinemática, despreciando los términos de orden superior, es:

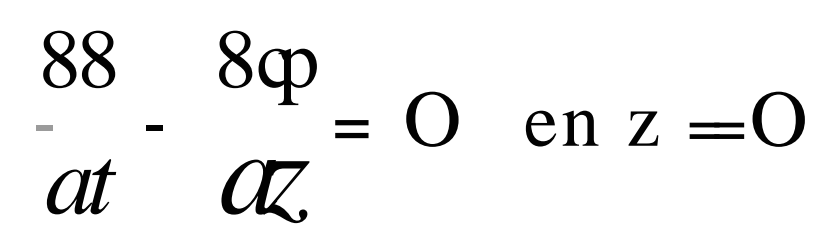

donde 8 es la altura de ola. La condición cinemática de contorno en la superficie libre controla el movimiento vertical de las partículas y garantiza que una partícula que se encuentre en la superficie libre permanezca en ella durante el movimiento. La condición dinámica se obtiene de aplicar Bemoulli en la superficie libre del líquido co11una presión 
atmosférica constante; considerando que la aceleración horizontal en la base del tanque actúa en la dirección $x, a x(t)$, se obtiene

$$
\frac{\partial \varphi}{\partial t} \quad g \delta \quad a_{x}(t) R \cos \theta \quad 0 \quad \text { en } z=O
$$

do11de g es la aceleración de la gravedad. La ventaja de usar los ejes coordenados seleccionados para formular la solución del problema es que las condiciones de frontera son homogéneas y que la aceleración del terreno aparece en la condición dinámica en forlna explícita. La altura de ola 8 se puede despejar de la ecuación (14)

$$
\delta-\frac{1}{\mathrm{~g}} \operatorname{ax} \quad \mathrm{a}_{\mathrm{x}}(\mathrm{t}) \mathrm{R} \cos \theta
$$

Derivando con respecto a t y sustituyendo en (13) se obtiene,

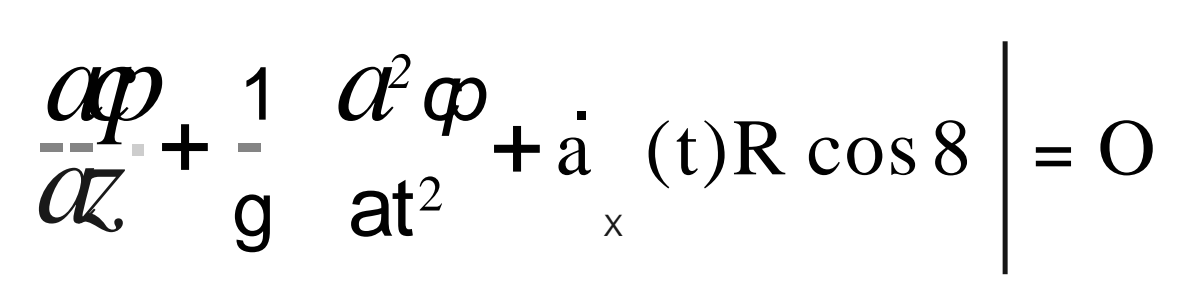

donde ${ }^{a} \mathrm{x}(\mathrm{t})$ es la derivada de la aceleración con respecto al tiempo. El potellcial de velocidad $\operatorname{cp}(\mathrm{R}, 8, \mathrm{z}, \mathrm{t})$, ecuación (1O), debe satisfacer las condiciones de frontera. Así para la condición en el fondo del tanque,

$$
\frac{\partial \varphi}{O R} \quad \mathrm{~F}(\mathrm{t})\left[\mathrm{A}_{1} \operatorname{sen}(\mathrm{m} \theta) \quad \mathrm{A}_{2} \cos (\mathrm{m} \theta)\right] \mathrm{C}_{\mathrm{m}} \mathrm{J}_{\mathrm{m}}(\mathrm{kR})\left[\mathrm{B}_{1} \mathrm{k} \exp (\mathrm{kz})-\mathrm{B}_{2} \mathrm{k} \exp (-\mathrm{kz})\right]
$$

igualando a cero y evaluando en $\mathrm{z}=\mathrm{h}$ se obtiene que

$$
\mathrm{B}_{1}=\mathrm{B}_{2} \exp (2 \mathrm{kh})
$$

Sustituyendo en la ecuación (10)

$$
\mathrm{cp}(\mathrm{R}, 8, \mathrm{z}, \mathrm{t})=\mathrm{F}(\mathrm{t})\left[\mathrm{A}_{1} \operatorname{sen}(\mathrm{m} 8)+\mathrm{A}_{2} \cos (\mathrm{m} 8) \mathrm{J} 2 \mathrm{~B}_{2} \exp (\mathrm{kh}) \cosh [\mathrm{k}(\mathrm{z}+\mathrm{h})] \mathrm{kmJm}(\mathrm{kR})\right.
$$

El potencial de velocidad debe satisfacer la condición dinámica en vibración libre en la superficie libre del líquido, es decir

$$
8=-\begin{array}{r|r}
1 & a p \\
-g & \text { at }
\end{array} \mid \text { en } \mathrm{z}=0
$$

Derivando la ecuación (19) con respecto a t, tomando la solución cosenoidal y sustituyéndola en (20) se tiene,

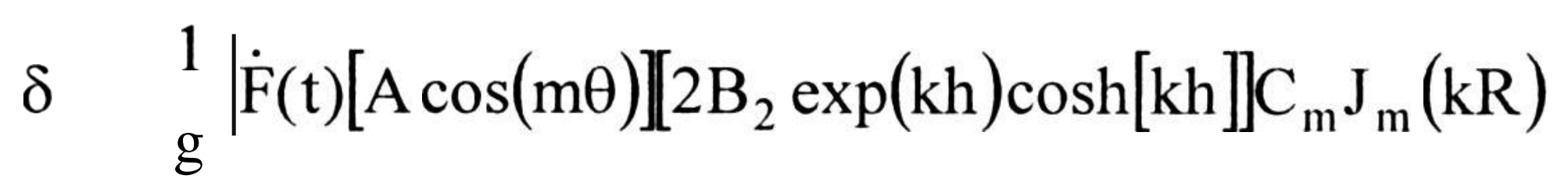


Considerando que la máxima amplitud del oleaje, $\mathrm{H}$, se presenta en las paredes del tanque, $\mathrm{R}=\mathrm{a}$, la expresión (21) se puede escribir

$$
\mathrm{H}=2 \mathrm{~B}_{2} \exp (\mathrm{kh}) \cosh [\mathrm{kh}] \mathrm{J}_{{ }_{1} \mathrm{n}}(\mathrm{ka}) \mathrm{D}
$$

de donde

$$
2 \mathrm{~B}_{2} \exp (\mathrm{kh}) \quad \mathrm{E}_{\mathrm{m}} \begin{gathered}
1 \\
\cosh \mathrm{kh}
\end{gathered} \frac{1}{\mathrm{~J}_{\mathrm{n}}(\mathrm{ka})}
$$

donde D y H están contenidas en Em.

Al sustituir la ecuación (22) en (21) se puede ver que la altura de ola, 8 , se puede escribir como:

$$
8(\mathrm{R}, 8, \mathrm{t})=1 \mathrm{t}^{\prime}(\mathrm{t}) \operatorname{sm}(\mathrm{R}, 8)
$$

es decir como el producto de una función dependiente de $t$, I $\left(\mathrm{V}_{\mathrm{t}}\right)$, y una función que representa la variación espacial de la ola,

$$
\mathrm{S}_{\mathrm{m}}(\mathrm{R}, \theta) \frac{\mathrm{J}_{\mathrm{m}}(\mathrm{kR})}{\mathrm{J}_{\mathrm{m}}(\mathrm{ka})} \cos (\mathrm{m} \theta)
$$

Sustituyendo la ecuación (22) en la (19) obtenemos,

$$
\varphi(\mathrm{R}, \theta, \mathrm{z}, \mathrm{t}) \quad \mathrm{F}(\mathrm{t}) \mathrm{E}_{\mathrm{m}} \cos (\mathrm{m} \theta) \frac{\mathrm{J}_{\mathrm{m}}(\mathrm{kR})}{\mathrm{Jm} \mathrm{ka}} \frac{\cosh [\mathrm{k}(\mathrm{z} h)}{\cosh \mathrm{kh}]}
$$

La ecuación (25) debe cumplir con la condición de frontera en lapared del tanque,

$$
\frac{\partial \varphi}{\partial R}=F(t) E_{m} \cos (m \theta) \frac{J_{m}^{\prime}(k a)}{J_{m}(k a)} \frac{\cosh [k(z+h)]}{\cosh [k h]} 0
$$

Para una solución diferente a la trivial se requiere que 1:n $(\mathrm{ka})=$ Olo cual se satisface para un número infinito de valores discretos de $\mathrm{k}$. Denotemos estos valores empleando doble subíndice, así kmn denota la n-ésima raíz de la derivada de la función de Bessel de primer tipo de m-ésimo orden., La solución general del potencial de velocidad se obtiene superponiendo todas las posibles soluciones:

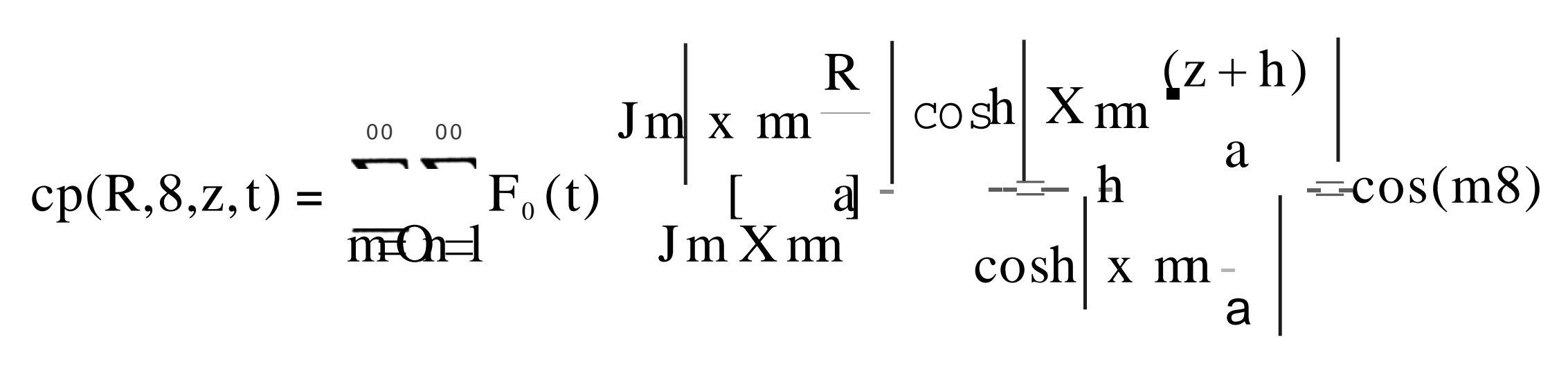


donde $X m_{11}=\mathrm{k}_{1}$ na es la raíz positiva de la primer derivada de la función de Bessel, $\mathrm{J} \quad[\mathrm{m} \mathbf{m}]=\mathrm{O}$. En tanques cilíndricos con paredes rígidas el modo fundamental de vibrar, en la dirección angular, se presenta en $\mathrm{m}=1$, por lo que la solución del potencial es:

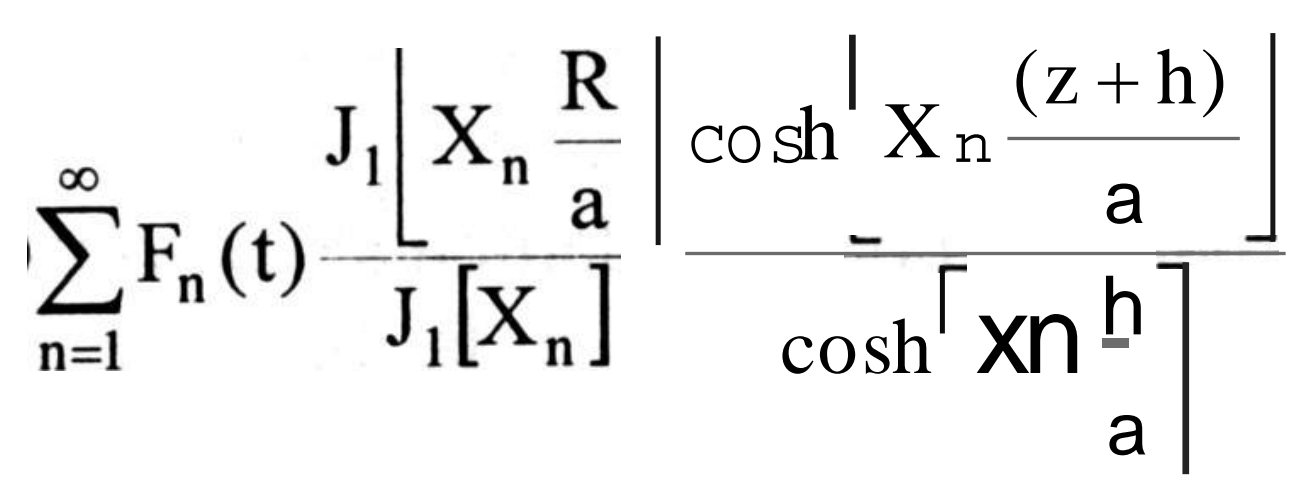

La frecuencia natural de vibrar de la superficie del líquido, $\mathbf{w}_{0}$, se obtiene de la ecuación (25) con $\mathrm{F}(\mathrm{t})$ = sen cot $\mathrm{y}$ haciendo uso de las condiciones de frontera en la superficie en vibración libre,

$$
\mathrm{w}_{\mathrm{n}}=\sqrt{\mathrm{gk}_{\mathrm{n}} \tanh \left[\mathrm{k}_{\mathrm{n}} \mathrm{h}\right]}
$$

donde $\mathrm{k}_{\mathrm{n}} \frac{\mathrm{X}_{\mathrm{n}}}{\mathrm{a}}$ Substituyendo el potencial de velocidad, $\varphi(\mathrm{R}, \theta, \mathrm{z}, \mathrm{t})$, en la expresión (16) se tiene

$$
\sum_{\mathrm{n}=1}^{\infty} \frac{\mathrm{J}_{1}\left\lfloor\mathrm{X}_{\mathrm{n}} \frac{\mathrm{R}}{\mathrm{a}} \mid\right.}{\mathrm{J}_{1}\left[\mathrm{X}_{\mathrm{n}}\right]} \mid \mathrm{F}_{0}(\mathrm{t})+\mathrm{w}_{0}^{2} \mathrm{~F}_{0}(\mathrm{t})+\operatorname{Rax}(\mathrm{t})=\mathrm{O}
$$

La solución para Fn(t) en (29) se puede obtener por medio de las series de Fourier-Bessel y haciendo uso de las propiedades de ortogonalidad de las funciones de Bessel,

$$
\left.\ddot{F}_{n}(t)+w_{n}^{2} F_{n}(t)=\frac{2 a}{1-X_{n}^{2}}\right]^{a_{x}}(t)
$$

Esta expresión es similar a la ecuación de movimiento no amortiguada de un oscilador simple con frecuencia natural igual a la frecuencia natural de vibrar del líquido sometido a una excitación que depende de la derivada de la aceleración sísmica del terreno.

\section{AMORTIGUAMIENTO VISCOSO}

Considerando la teoría de oleaje pequeño, el movimiento del fluido es esencialmente irrotacional excepto cerca de las fronteras sólidas del tanque (Mei y Liu, 1973). La teoría del potencial descrita no predice disipación de energía. Sin embargo, resultados experimentales en tanques para almacenamiento, con superficie libre, muestran claramente que existe un comportamiento amortiguado en la respuesta del líquido (Case y Parkinson, 1957); hay disipación de energía relacionada con el amortiguamiento en el movimiento del fluido. La disipación de energía se produce en la capa límite cerca de las paredes y el fondo del tanque, y en la capa límite cerca de la superficie libre del líquido, farmándose un 
menisco entre el líquido y la pared. La cantidad de energía disipada depende de la forma del tanque, de la rugosidad de las paredes y de la viscosidad del líquido.

En pruebas experimental es de tanques rectangulares almacenando agua, se han medido decrementos logarítmicos equivalentes a coeficientes de amortiguamiento crítico del orden de $0.35 \%$ (Keulegan, 1958). En pruebas de vibración ambiental de tanques cilíndricos se ha ton 1ado un valor de $\mathrm{s}=0.5 \%$ para el modo ft1ndamental de vibrar del líquido (Housner $\mathrm{y}$ Haroun, 1980; 1983). Si el tanque está fijo a la base se recomienda un factor de amortiguamiento del sistema tanque-líquido del $\mathrm{s}=2 \%$, en su modo fundamental (Veletsos y Shivakumar, 1997) y de $\mathrm{s}=0.5 \%$ en el caso de líquidos estratificados (Veletsos y Shivakumar, 1993). Los códigos para el diseño de tanques cilíndricos de almacenamiento proponen un valor de $\mathrm{s}=0.5 \%$ para el modo fundamental de vibrar del líquido. En el análisis de estabilidad del movimiento del líquido bajo rotación y traslación del tanque, se ha propuesto como condición más desfavorable considerar que el amortiguamiento es nulo (Rogge y Weiss, 1966).

Para simular el efecto del amortiguamiento viscoso en la teoría del potencial para tanques cuadrados, Faltinsen (1978) introdujo un término ficticio para la viscosidad en la ecuación de movimiento y propuso un coeficiente de amortiguamiento crítico del $5 \%$ para el primer modo de vibrar del líquido. Con el fín de tomar en cuenta el efecto de disipación de energía, en este estudio se incluyó un amortiguamiento en la ecuación (30) de manera análoga al caso de la ecuación de movimiento de un oscilador con amortiguamiento vïscoso,

$$
\left.\ddot{\mathrm{F}}_{\mathrm{n}}(\mathrm{t})+2 \xi_{\mathrm{n}} \mathrm{w}_{\mathrm{n}} \dot{\mathrm{F}}_{\mathrm{n}}(\mathrm{t})+\mathrm{w}_{n}^{2} \mathrm{~F}_{\mathrm{n}}(\mathrm{t})=\frac{2 \mathrm{a}}{\left[1-\mathrm{X}_{\mathrm{n}}^{2}\right.}\right]^{\mathrm{a}}{ }_{\mathrm{x}}(\mathrm{t})
$$

\section{PROCEDIMIENTO DE ANÁLISIS}

Una vez que se conoce el potencial $\Varangle$ (ecuación (27)) la altura de ola en la superficie libre del líquido, z=O, se calcula con la ecuación (15)

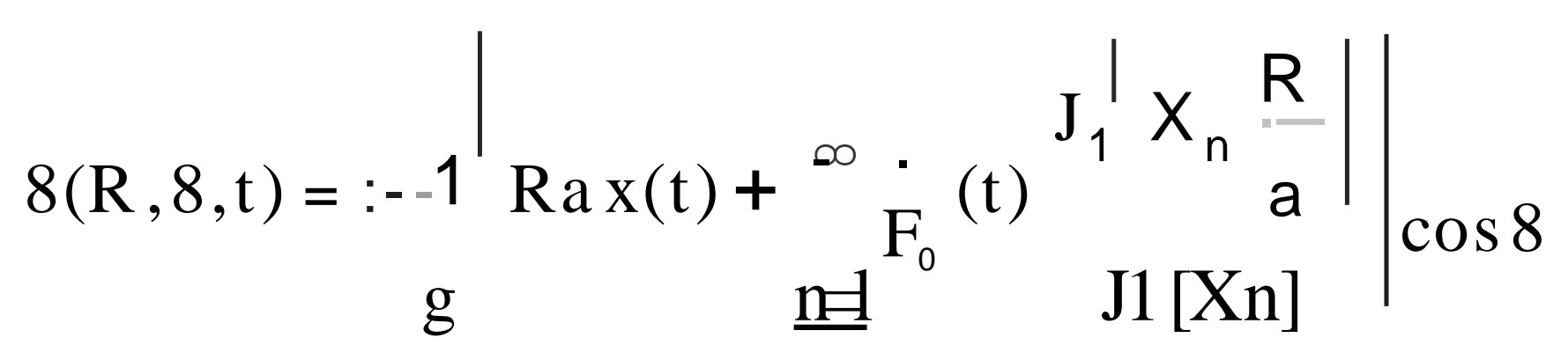

Despreciando los términos de orden superior, la ecuación de Bemoulli se puede escribir como:

$$
\alpha
$$$$
\mathrm{p}
$$

donde Ph es la presión hidráulica. Despejando Phde esta ecuación, se tiene 


$$
\mathrm{Ph}(\mathrm{R}, 8, \mathrm{z}, \mathrm{t})=-\mathrm{p}|\mathrm{gz}+\mathrm{ax}(\mathrm{t}) \mathrm{R} \cos 8+\underset{8 \mathrm{t}}{\operatorname{aqp}}|
$$

Se puede notar que el primer término en el lado derecho de la ecuación (34) representa la presión hidrostática, -pgz ; los siguientes términos representan la presión hidrodinámica producto de la excitación horizontal del terreno. En función del potencial de velocidad, la presión hidráulica sobre las paredes del tanque, se calcula con:

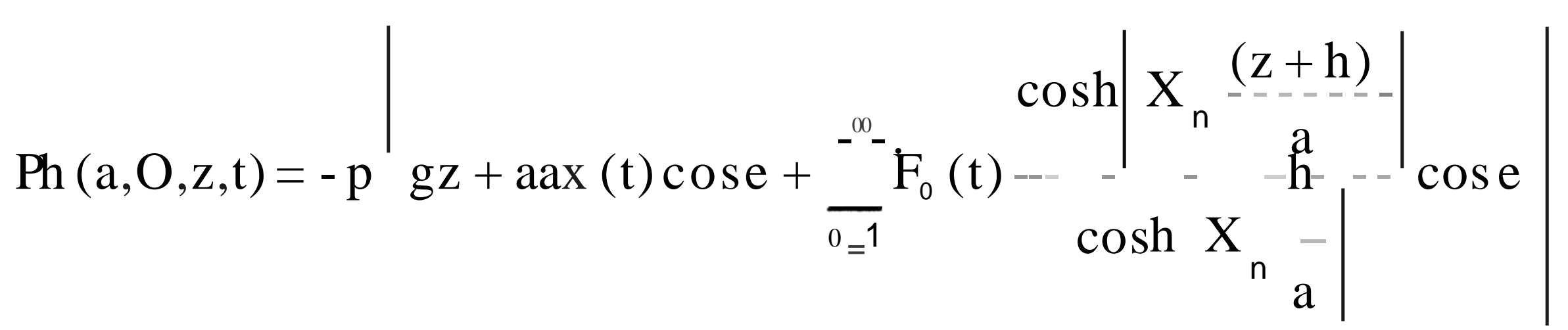

La fuerza cortante en la pared del tanque se obtiene integrando la presión hidrodinámica en la altura,

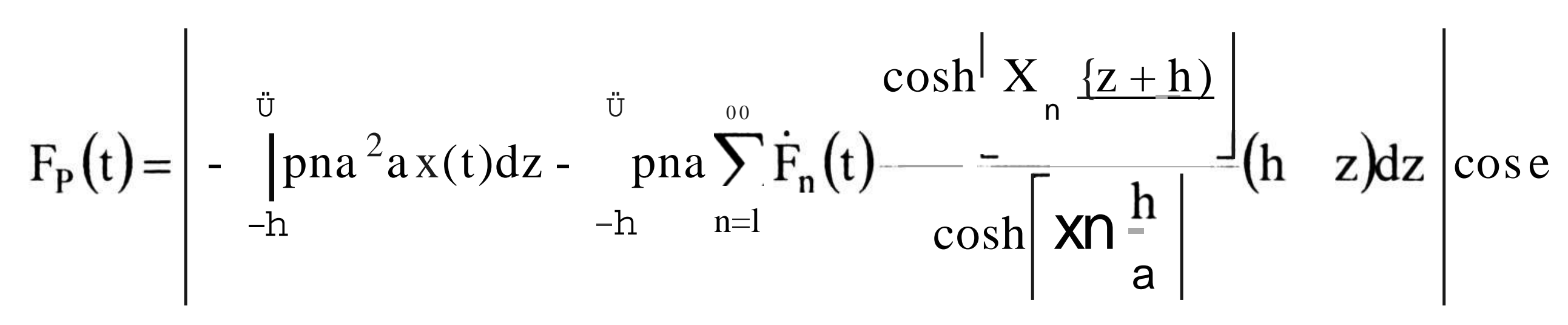

es decir

$$
F_{p}(t)=-\left(\rho \pi a^{2}\right)\left|a_{x}(t) h+\sum_{n=1}^{\infty} \dot{F}_{n}(t) \frac{1}{X_{n}} \tanh \left\lfloor X_{n} \frac{h}{a}\right\rfloor\right| \cos \theta
$$

El momento de volteo en la base de la pared del tanque se obtiene de:

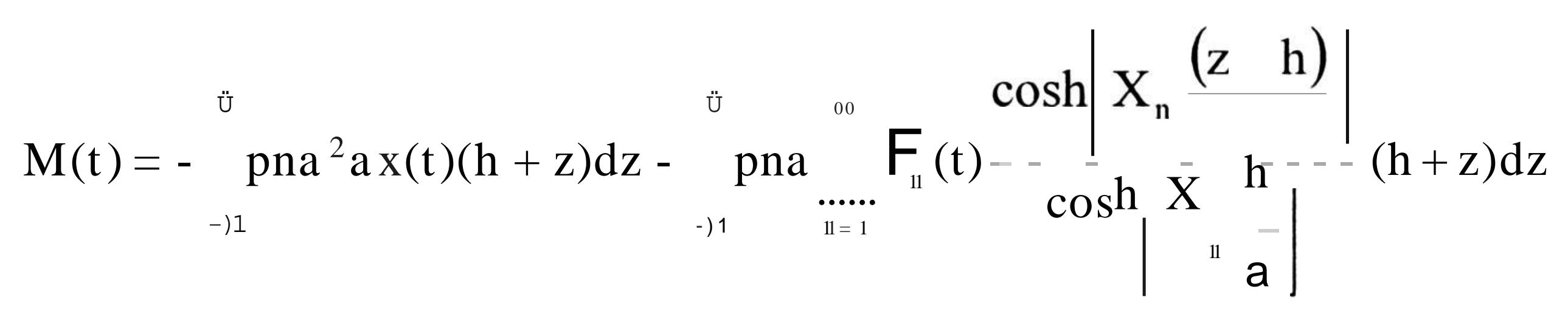

de do11de

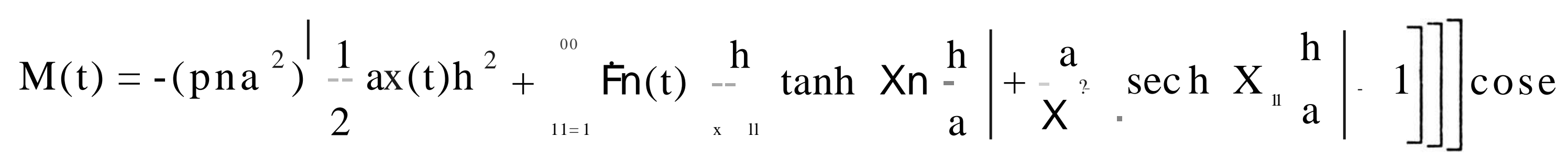

\section{BIDIRECCIONALIDAD DE LA EXCITACIÓN}

Una vez que se han resuelto las ecuaciones linealizadas que gobiernan el comportamiento diná1nico de un tanque cilíndrico sometido a una excitación sísmica horizontal en la base, se puede hacer uso del principio de superposición para obtener la solución del problema 
dinámico ante dos componentes horizontales ortogonales actuando simultáneamente en su base. Sea ay(t) la componente de la aceleración del terreno actuando en la dirección y. La altura de ola debida a esta componente de la excitación se calcula con:

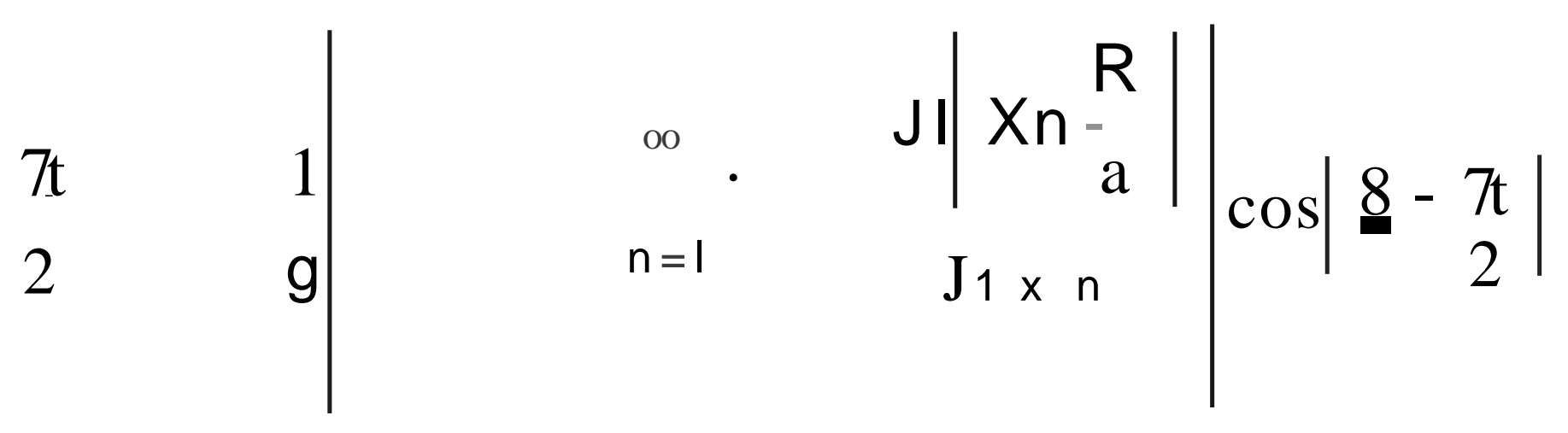

y la amplitud total considerando ambas componentes horizontales es:

$$
\delta_{\mathrm{T}}(\mathrm{R}, \theta, \mathrm{t})=\delta_{\mathrm{x}}(\mathrm{R}, \theta, \mathrm{t})+\delta_{\mathrm{y}}\left(\mathrm{R}, \theta-\frac{\pi}{2}, \mathrm{t}\right)
$$

Las presiones hidráulicas totales en las paredes del tanque se puede11calcular como sigue,

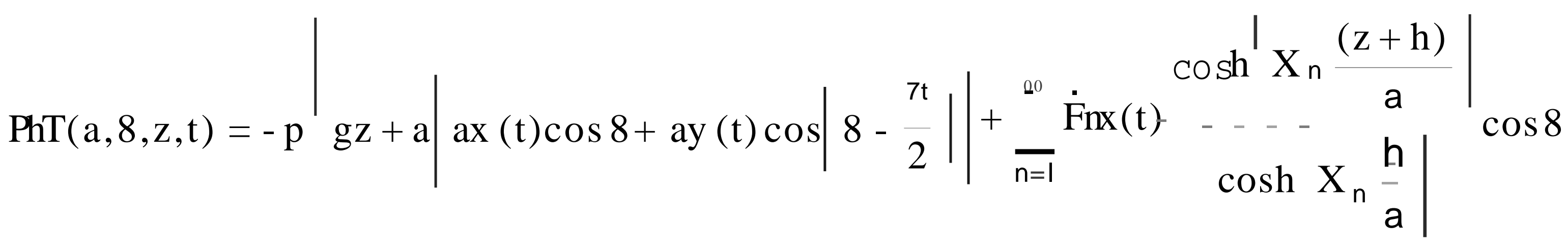

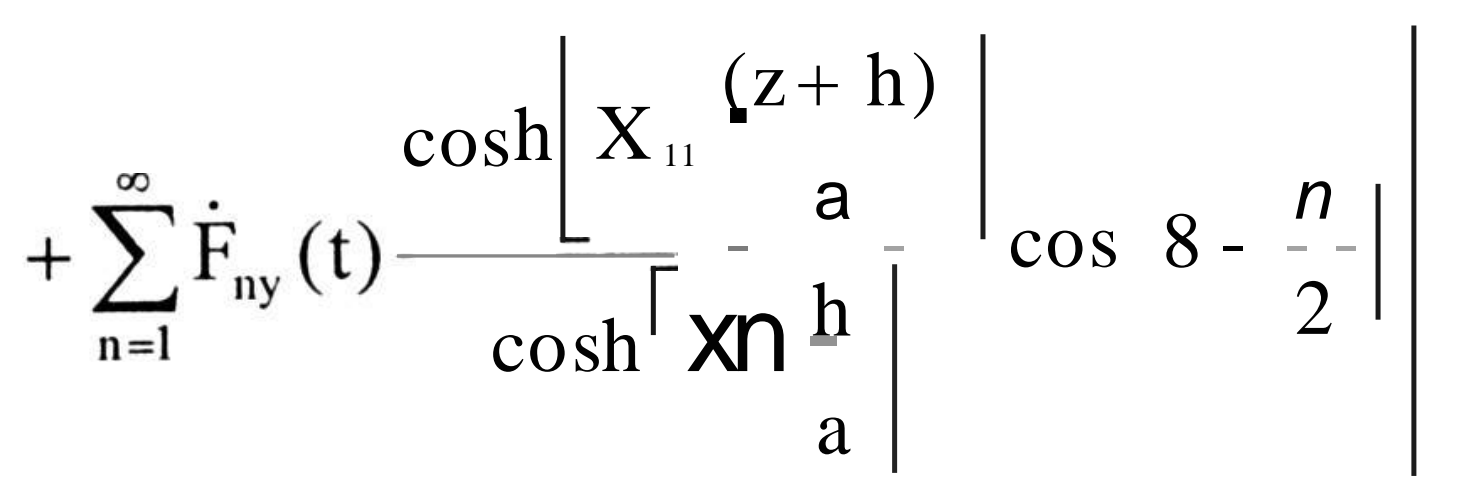

De la misma manera se pueden escribir las ecuaciones para el cálculo de las fuerzas cortantes y momentos de volteo totales con base en las ecuaciones (36) y (37).

\section{COMPONENTE VERTICAL DE LAEXCITACION}

Se cree que las fallas por pande9 tipo "pata de elefante" en tanques cilíndricos ubicados en zonas epicentrales se producen principalmente por la componente vertical de la aceleración sísmica del terreno (duanquing, 1984). Los modelos matemáticos que se han utilizado para investigar la respuesta de tanques cilíndricos ante excitaciones sísmicas verticales no consideran el oleaje en la superficie libre del líquido. Estos modelos se basan en pruebas realizadas en modelos a escala en las que no se ha presentado oleaje debido a la excitación vertical. Si el fluido contenido en el tanque es incompresible y no viscoso en todo punto del volumen, Q, existe un potencial de velocidad,$\backslash>$, que satisface la ecuación de Laplace, $\mathrm{V}^{2} \triangleleft=\mathrm{O}$. Consideramos únicamente la componente vertical de la excitación del terreno, $\mathrm{a}_{2}(\mathrm{t})$ y suponemos alturas de ola pequeñas; las condiciones de frontera con respecto a un eje coordenado relativo son:

$$
\begin{aligned}
& 8 \triangleleft>\mathrm{O} \text { en } \mathrm{R}= \pm \mathrm{a} \\
& 8 \mathrm{R}
\end{aligned}
$$




$$
\begin{aligned}
& 8 \nrightarrow \\
& 8 \mathrm{z}
\end{aligned}
$$

La condición de contorno en el fondo del tanque, ecuación (42), garantiza que la velocidad vertical de una partícula de fluido que limita con la frontera sólida del fondo es igual a la componente vertical de la velocidad del terreno. En la superficie libre del líquido la condición cinemática es,

$$
88 \stackrel{8 \triangleleft}{8} \stackrel{8}{-}-\mathrm{O}^{\prime} \mathrm{Z}+\mathrm{V}_{2}(\mathrm{t})=\mathrm{O} \text { en } \mathrm{z}=\mathrm{O}
$$

La expresión (43) tiene un claro signíficado físico: el oleaje en la superficie libre se propaga con una velocidad relativa del líquido igual a $\left|\underset{8 \mathrm{Z}}{8 \triangleleft}-\mathrm{V}_{2}(\mathrm{t})\right|$. Por otro lado, la condición dinámica en la superficie libre del líquido es:

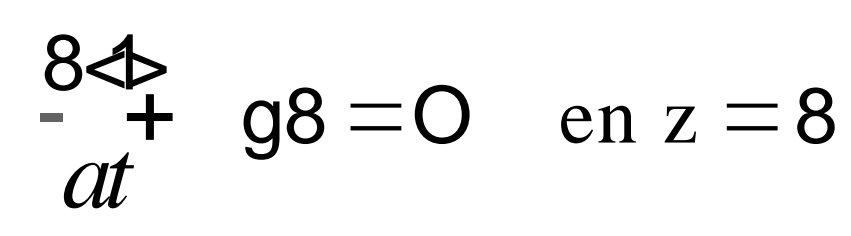

En las ecuaciones (41) a (44) el potencial de velocidad, $\$$, se puede escribir como,

$$
\Phi=\varphi+\mathrm{V}_{\mathrm{z}}(\mathrm{t}) \mathrm{Z}
$$

El primer término en el lado derecho de 45), $\varsigma$, es el potencial de velocidad relativo al sistema coordenado relativo y soluciona las tres primeras ecuaciones de las condiciones de frontera en su forma homogénea; el segundo térrnino, soluciona las condiciones de frontera en su forma particular. La solución para el potencial $ৎ$ se puede escribir entonces como

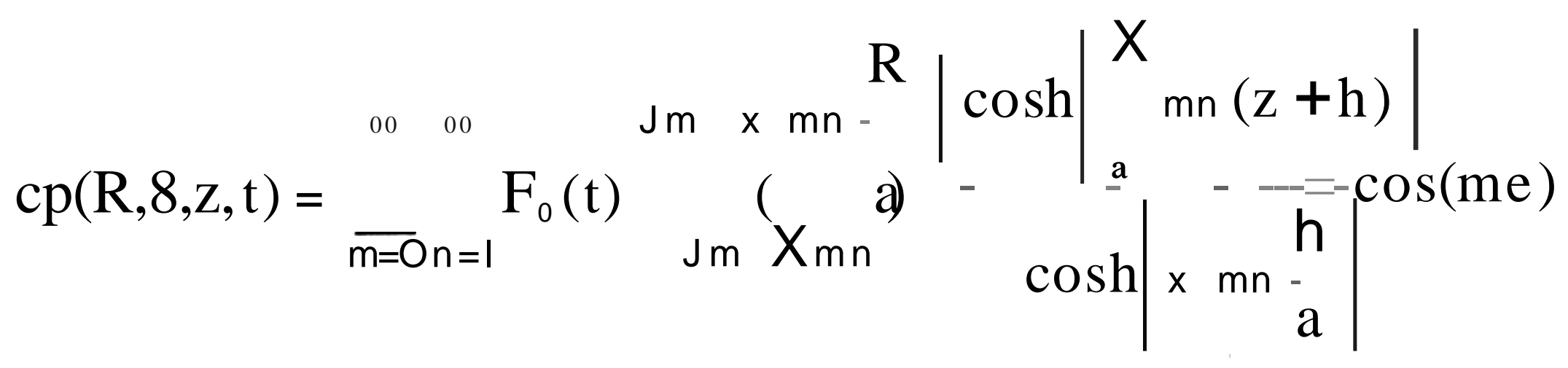

donde $\mathrm{X}_{\mathrm{nn}}=\mathrm{k} \ln 1 \mathrm{a}$ son las rtlíces positivas de la función de Bessel, $\mathrm{J}_{\mathrm{h}}\left(\mathrm{X}_{\mathrm{n} \mathbb{1}}\right)=\mathrm{O}, \mathrm{y}$ la frecuencia de vibrar en la sup rficie libre del líquido es:

$$
\mathrm{w}_{\mathrm{mn}}^{2}=\mathrm{gk}_{\mathrm{mn}} \tanh \left[\mathrm{k}_{\mathrm{mn}} \mathrm{h}\right]
$$

Pruebas experimentales muestran que la componente vertical de la aceleración excita principalmente los modos simétricos del tanque, es decir $\mathrm{m}=\mathrm{O}$; en tal caso el problema dinámico se conoce como problema de Faraday. De las ecuaciones (23) y (24), la altura de ola es de la forina: 


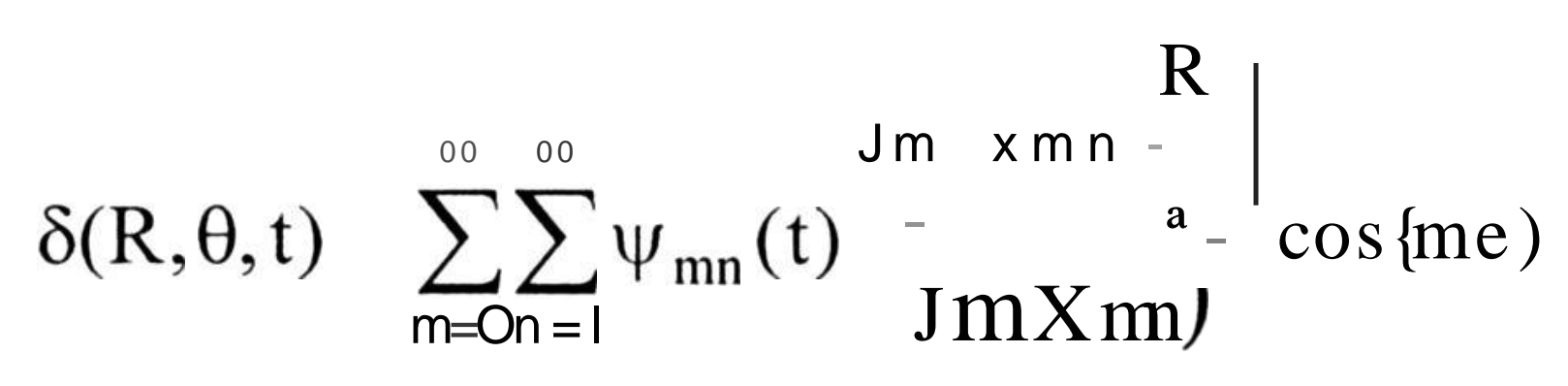

Sustituyendo (46) y (48) en las condiciones de frontera (43) y (44), se puede demostrar que la ecuación de movimiento del sistema es:

$$
\ddot{\psi}_{m n}(\mathrm{t})+2 \xi_{m n} \mathrm{w}_{m n} \dot{\psi}_{m n}(\mathrm{t})+\left[\mathrm{w}_{m n}^{2}+\mathrm{p}_{m n} \mathrm{a}_{\mathrm{Z}}(\mathrm{t})\right] \psi_{m n}(\mathrm{t})=0
$$

donde Pim $=k \operatorname{mtanh}\left[\mathrm{k}_{11} \mathrm{~h}\right]$. La ecuación (49) se conoce como ecuación de Hill si $\mathrm{a}_{2}(\mathrm{t})$ es una función periódica; si $\mathrm{a}_{2}(\mathrm{t})$ es una función senoidal entonces se conoce como la ecuación de Mathieu.

Las presiones hidráulicas en las paredes del tanque, considerando que existe únicamente la componente vertical de la aceleración del terreno, se calculan estableciendo la ecuación de Bernoulli en la superficie libre del líquido,

$$
P_{h}=-\rho\left[g z+a_{z}(t) z\right]
$$

es decir, la presión hidráulica es la suma de la presión hidrostática, -pgz, más la presión hidrodinámica debida a la contribución de la componente vertical de la excitación: - $\mathrm{a}_{2}(\mathrm{t}) \mathrm{z}$. Se puede observar que las presiones hidrodinámicas debidas a la componente vertical de la aceleración del terreno varían en forma lineal, siendo cero en la superficie libre del líquido $(\mathrm{z}=\mathrm{O})$ y máximas en la base del tanque $(\mathrm{z}=-\mathrm{h})$. Dependiendo del valor de la amplitud de la aceleración vertical del terreno, la presión hidrodinámica puede ser de igual o mayor magnitud que la presión hidrostática.

\section{EJEMPLO DE APLICACIÓN}

Consideremos un tanque cilíndrico con diámetro igual a $10 \mathrm{~m}$ y altura del líquido de 2.50 $\mathrm{m}$. El peso volumétrico del líquido contenido en el tanque es $\mathrm{y}=1 \mathrm{t} / \mathrm{m}^{3}$ y su densidad $\mathrm{p}=0.1019 \mathrm{ts}^{2} / \mathrm{m}^{4}$. La Tabla 1 lista las primeras 9 frecuencias y periodos modales de la superficie libre del líquido.

El tanque se sometió a 1 as componentes de aceleración NS y EW registradas durante el sismo del 19 de septiembre de 1985 en las estaciones de Ciudad Universitaria (CU), Viveros (VIV), Secretaria de Comunicaciones y Transporte (SCT), Central de Abastos (CA), Tacubaya (TACY) y Caleta de Campos (CALETA). La Figura 4 muestra las historias de altura de ola en la pared del tanque para cada registro sísmico. En general, las alturas de ola máximas producidas por los movimientos sísmicos son menores que $35 \mathrm{~cm}$; sin embargo, en el caso del registro SCT, las componentes NS y EW generan alturas de ola de $61 \mathrm{~cm}$ y de $95 \mathrm{~cm}$, respectivamente. Es de interés el caso del registro en la estación CA: para ambas componentes se producen alturas de ola mayores que la altura total del tanque. La Figura 5 muestra la historia de respuesta del oleaje en la superficie libre del tanque 
sometido al registro de CA (EW). Se puede observar el incremento excesivo de la respuesta por encima de las paredes del tanque. Estos resultados sugieren que se presenta cierto fenómeno de resonancia y la altura de ola no se puede predecir correctamente con la solución lineal.

Tabla 1 Frecuencias y periodos de vibrar del tanque del ejemplo de aplicación

\begin{tabular}{|c|c|c|}
\hline Modo & Frecuencia $(\mathrm{rad} / \mathrm{s})$ & Periodo $(\mathrm{s})$ \\
\hline 1 & 1.6196 & 3.9794 \\
\hline 2 & 3.2186 & 1.9521 \\
\hline 3 & 4.0917 & 1.5356 \\
\hline 4 & 4.7924 & 1.3111 \\
\hline 5 & 5.4002 & 1.1635 \\
\hline 6 & 5.9453 & 1.0568 \\
\hline 7 & 6.4440 & 0.9751 \\
\hline 8 & 6.9064 & 0.9098 \\
\hline 9 & 7.3397 & 0.8561 \\
\hline
\end{tabular}

La Tabla 2 muestra las altura.s de ola máximas, obtenidas para cada dirección del movimiento, considerando el modo fundamental y los primeros nueve modos de vibración; así como los incrementos porcentuales de respuesta. Se observa que los modos superiores de vibrar tienen una contribución importante en la respuesta sísmica. La contribución de los modos superiores no es independiente de las características de la excitación. En el caso de

Tabla 2 Altura de ola máxima para el ejemplo de aplicación

\begin{tabular}{|l|c|c|c|}
\hline \multirow{2}{*}{ Movimiento } & \multicolumn{2}{|c|}{ Altura de ola en cm } & \multirow{2}{*}{} \\
\cline { 2 - 3 } & Fundamental & 9 modos & \multirow{2}{*}{ Inc. \% } \\
& $1=0.5 \%$ & $\mathrm{n}=0.5 \%$ & \\
\hline CU.NS & 11.55 & 19.14 & 66 \\
\hline CU.EW & 21.39 & 25.67 & 20 \\
\hline ,vIV.NS & 17.86 & 21.78 & 22 \\
\hline ,vIV.EW & 26.56 & 31.25 & 18 \\
\hline SCT.NS & 33.27 & 61.10 & 84 \\
\hline SCT.EW & 66.75 & 94.60 & 42 \\
\hline CA.NS & 277.17 & 272.82 & --- \\
\hline CA.EW & 363.60 & 359.95 & --- \\
\hline$\ldots$ ACY.NS & 23.37 & 26.37 & 13 \\
\hline ·ACY.EW & 31.32 & 32.10 & 3 \\
\hline CALETA.NS & 18.56 & 16.38 & -13 \\
\hline CALETA.EW & 24.88 & 27.16 & 10 \\
\hline
\end{tabular}


CU.NS
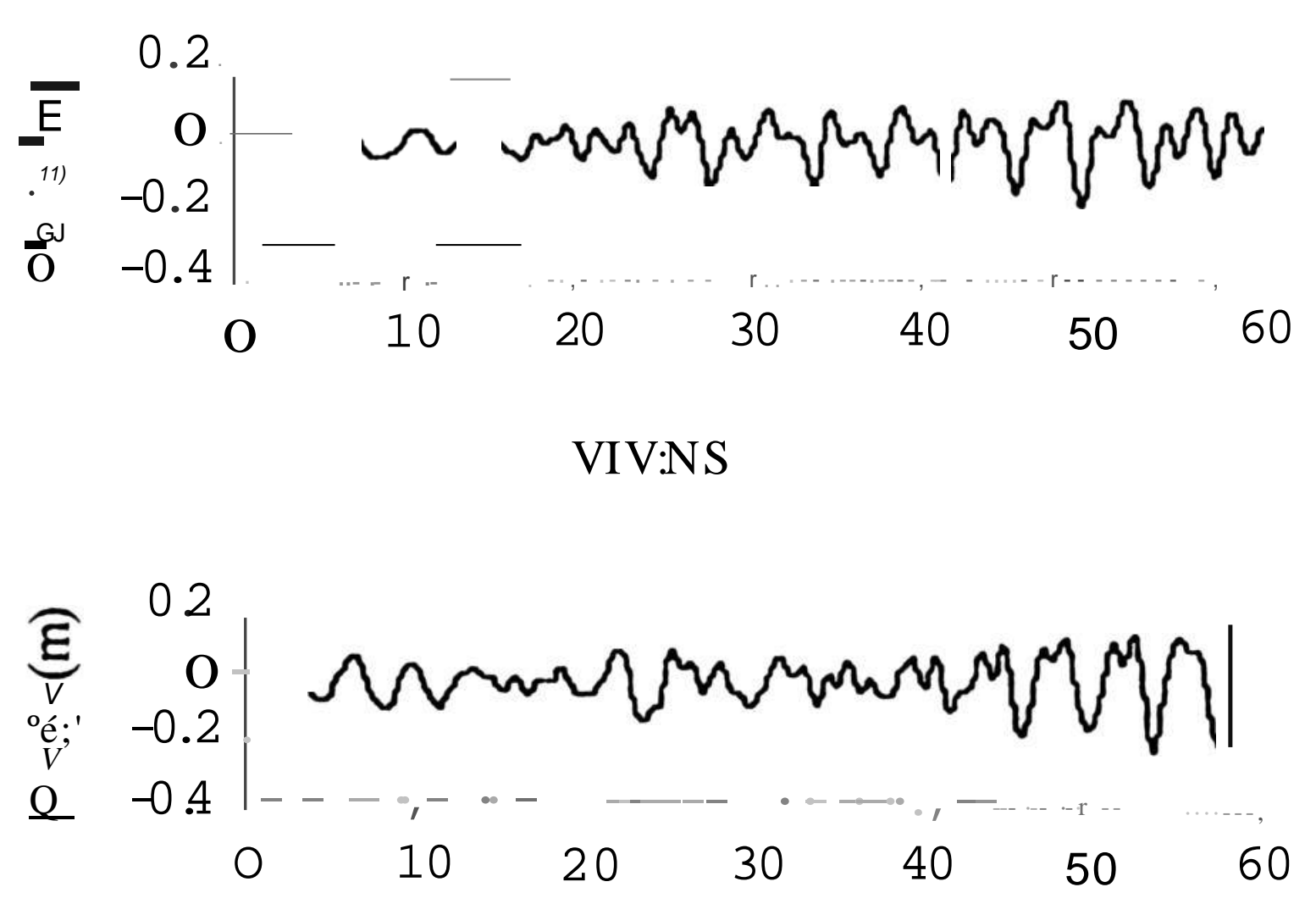

scr.Ns

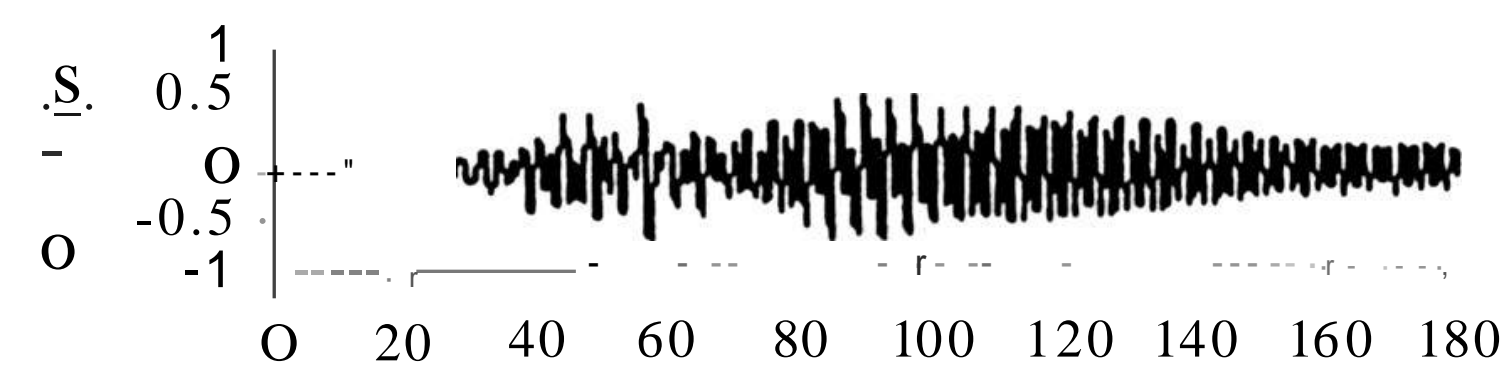

C, I.NS

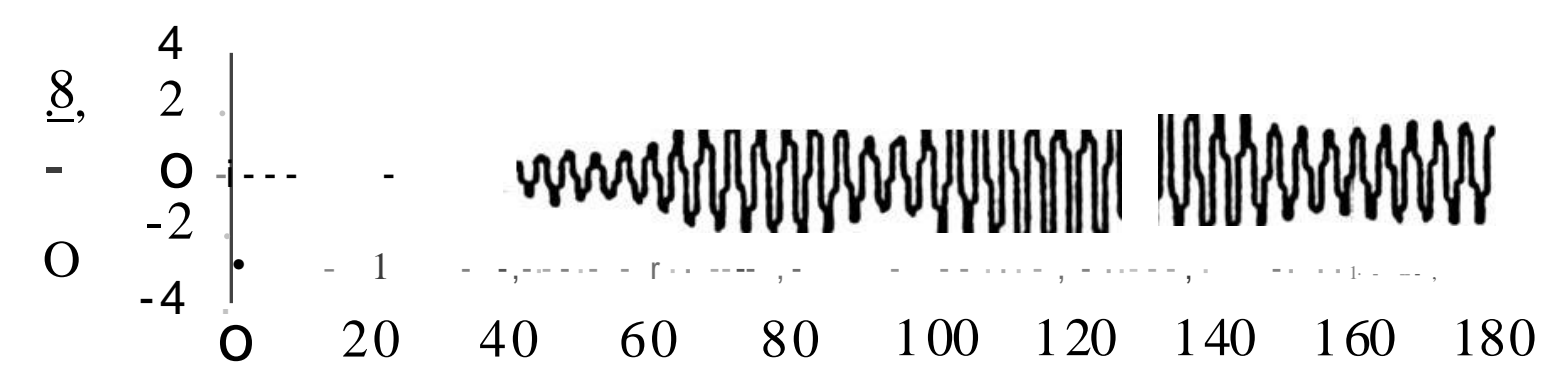

$\cdot 1 \cdot A C Y . N S$

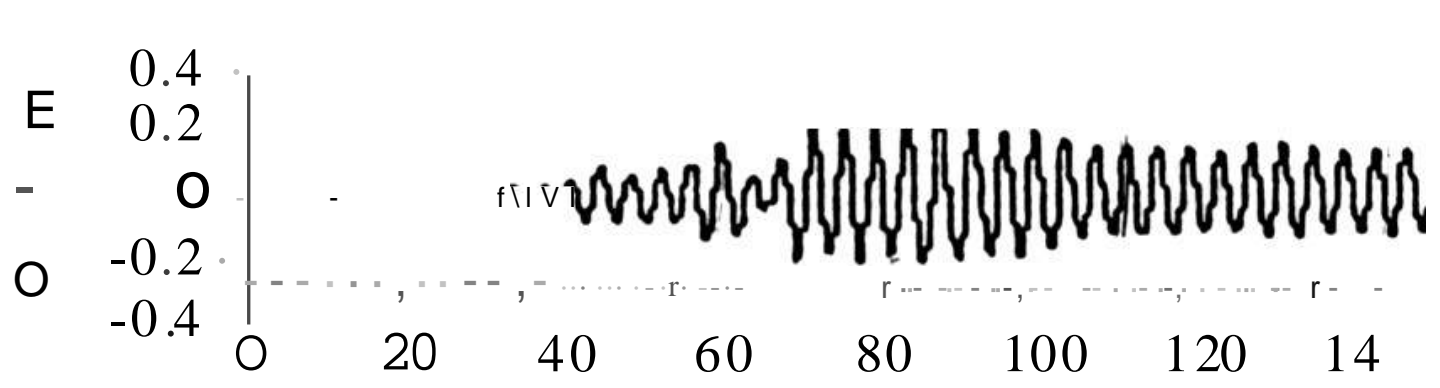

CALET $\Lambda . N S$

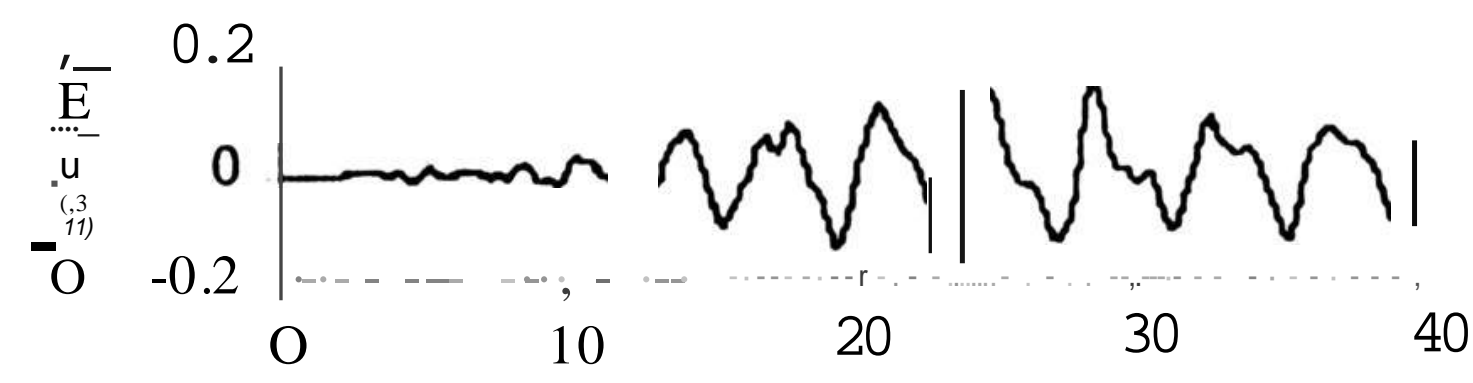

CU.EW

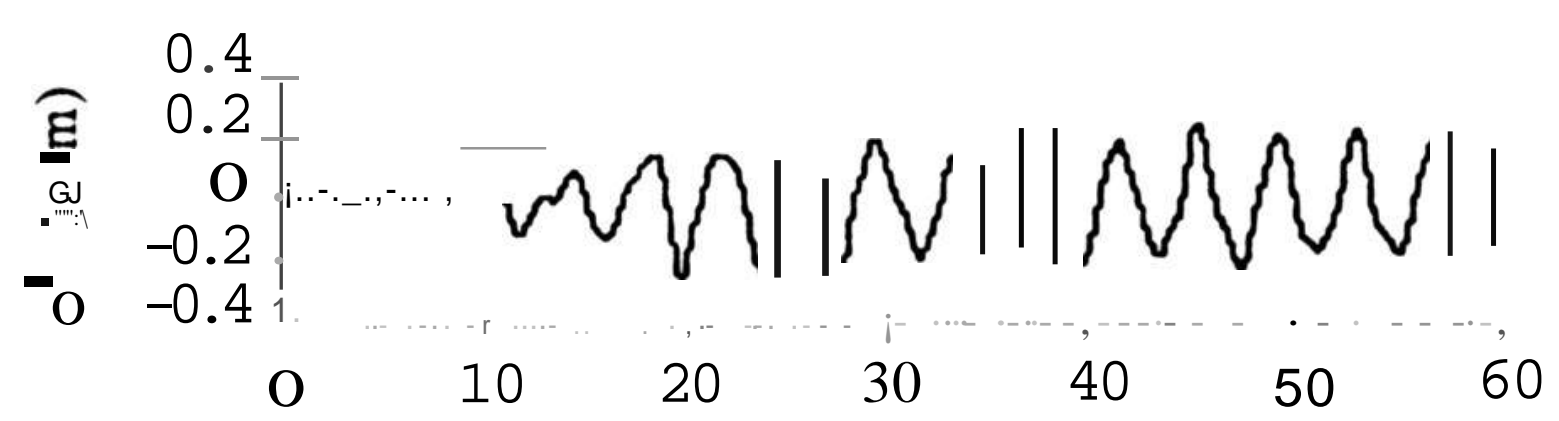

VIV.EW

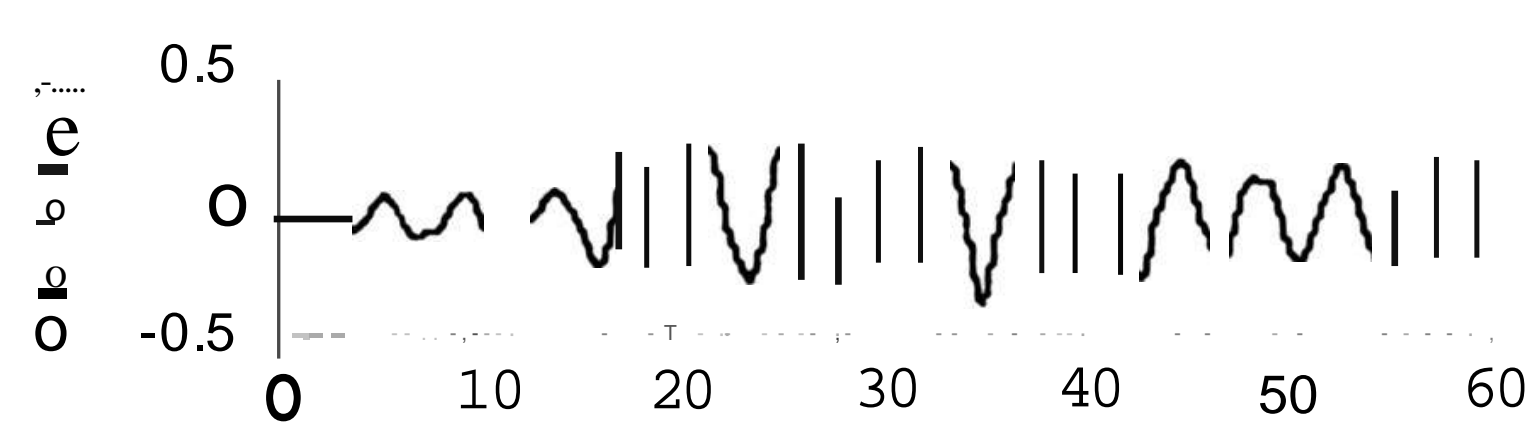

s e $\cdot$ i . EW

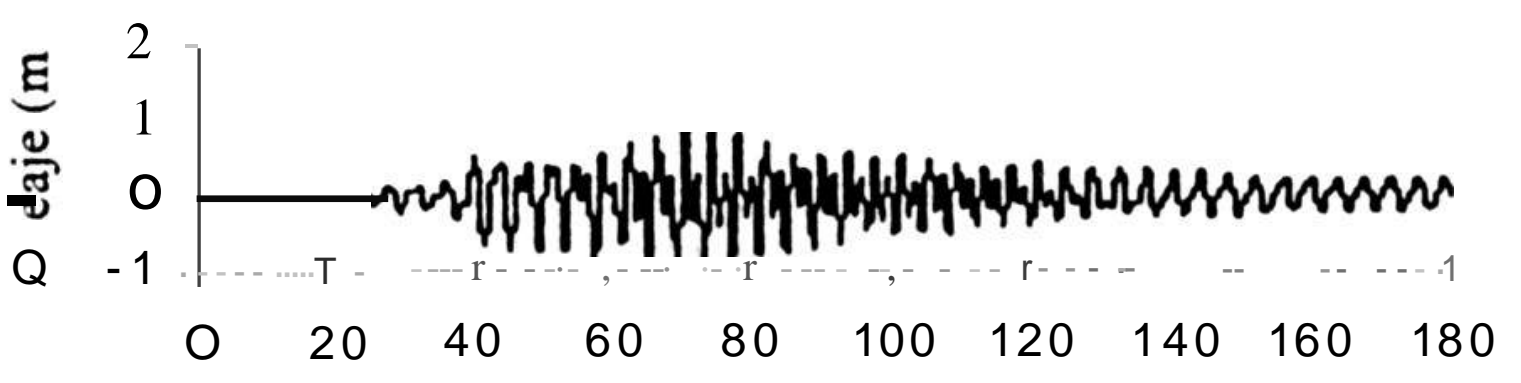

T AC) '.I. IV

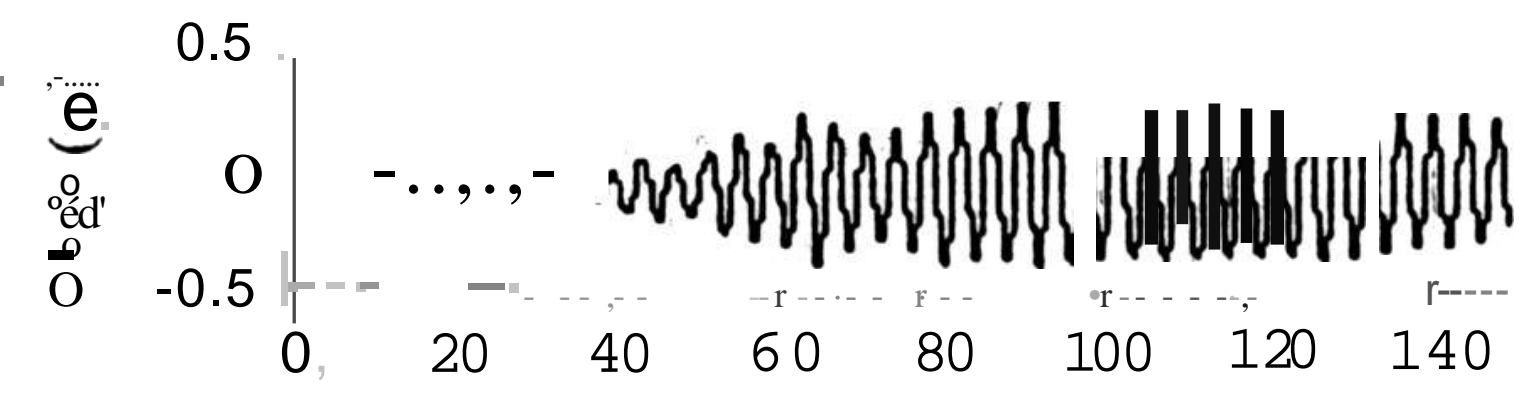

CAi.JETA.EW

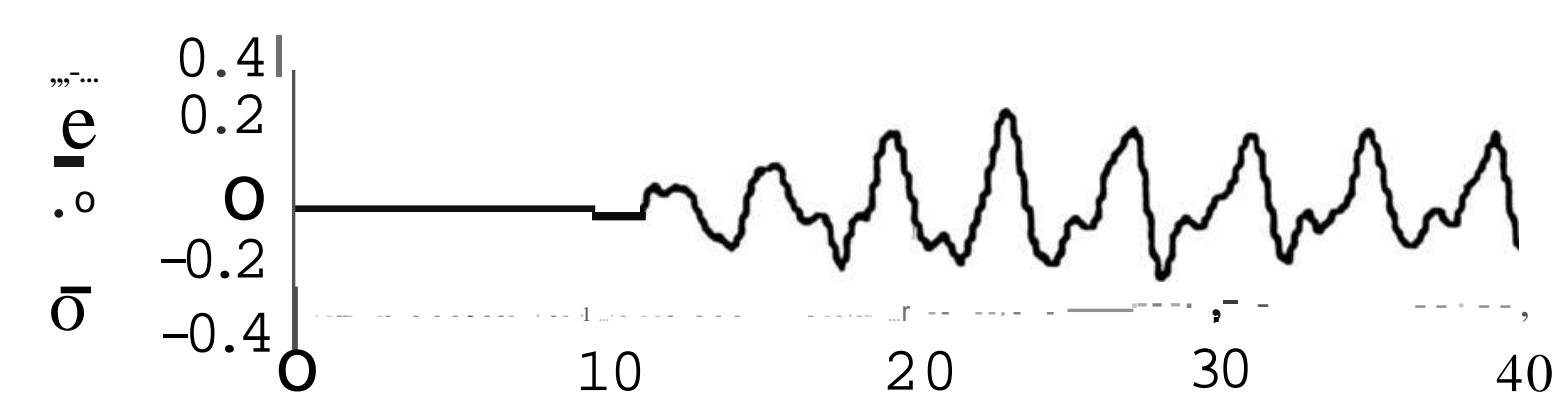

Fig 4. Historias de altura de ola en la pared del tanque 

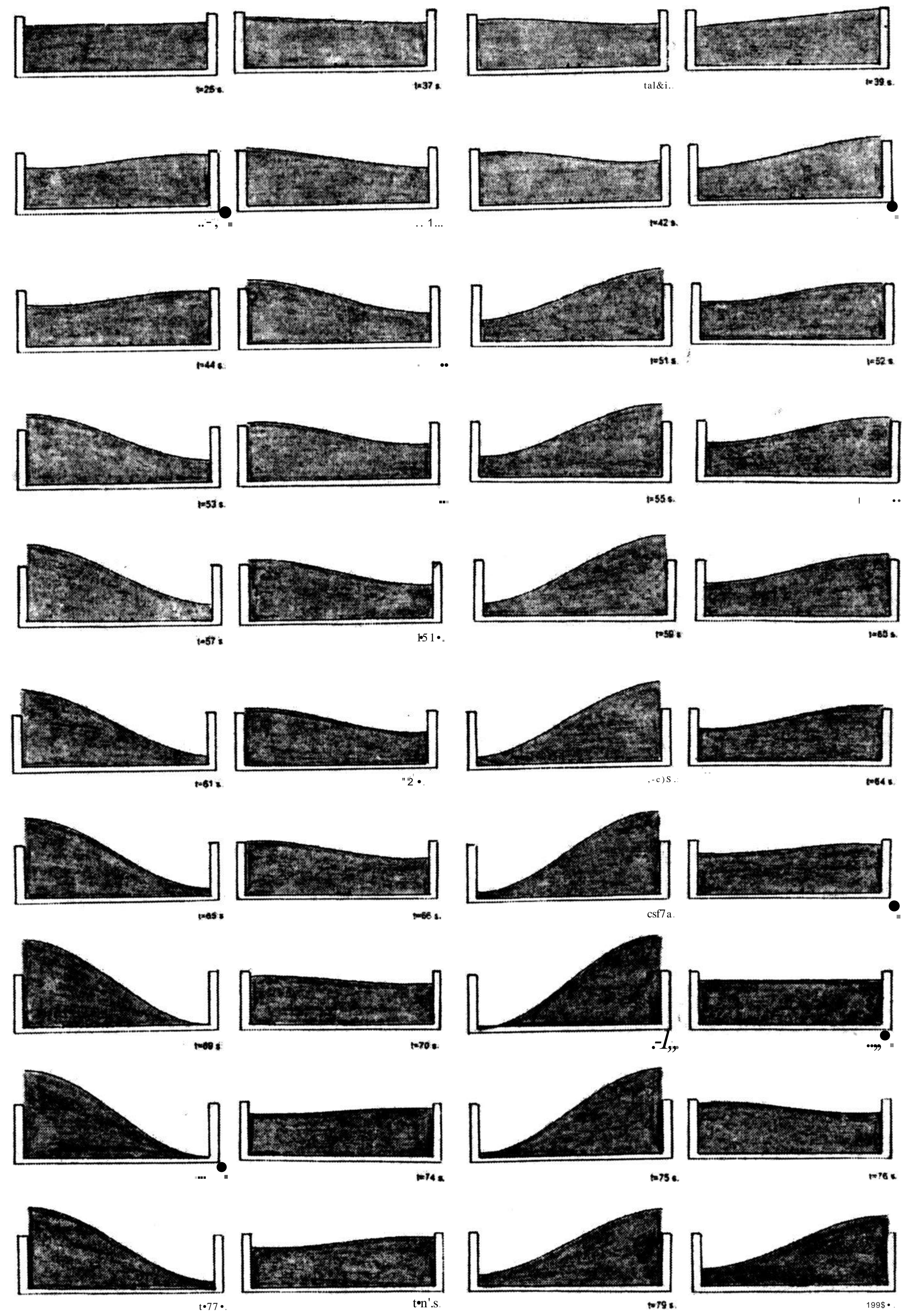

Fig 5. Respuesta de oleaje ante la excitación sísmica CA(EW) 
la componente NS del registro SCT, los modos superiores pueden incrementar la respuesta en $840 / 0$; dicha contribución es de $42 \%$ cuando se trata de la componente EW del mismo registro. Para el registro $\mathrm{CU}$, componente NS, el incremento por contribución de modos superiores $(66 \%)$ es más de tres veces el correspondiente a la componente EW del mismo registro (20\%). Despreciar esta contribució en el análisis de respuesta puede resultar e11 esfuerzos de diseño considerablemente menores que los actuantes en las paredes del tanque. Obsérvese que la contribución de los modos superiores puede ser negativa, como sucede con CALETA; estas obse1-vaciones se han reportado en otros estudios (Chen et al., 1996).

Se calcularon las fuerzas cortantes máximas en la pared del tal1que para cada componente horizontal de los movimientos sísmicos registrados. Primero se consideró que sólo actúa un componente horizontal de excitación en la base y luego se consideraron simultáneamente las dos componentes horizontales ortogonales. La Tabla 3 presenta las fuerzas corta11tes máximas obtenidas. En la cuarta columna se presenta el incremento porcentual entre an 1 bas respuestas y por último la dirección en la que se presenta la máxima respuesta ante dos excitaciones simultáneas definida por el ángulo 8; en este análisis se consideró que la componente NS actúa en la dirección $8=0^{\circ}$. Supongamos que el analista procede a realizar el diseño con base en la componente de respuesta con mayor demanda de esfuerzos. Se observa que en el caso de los registros CU, SCT y CALETA, el incremento de respuesta por bidireccionalidad es menor al 10\%. Sin embargo en el caso del registro Viveros el incremento de respuesta por acción de ambas colnponentes horizontales de la excitación es del orden del $45 \%$. Errores importantes de subestimación de la respuesta se obtienen en este caso si no se considera la bidireccionalidad de la excitación. Es intel-esante observar que las demandas de fuerza corta11te en cada componente son aproximadamente iguales y que el ángulo en el que se produce la respuesta máxima ante la acción simultánea de ambas componentes es casi de $45^{\circ}$ en el cuarto cuadrante. En caso de analizar la respuesta ante sólo una componente de excitació11, se podría incurrir en errores graves. Por ejemp lo, para la componente NS del registro SCT el increme11to de respuesta por efectos de bidireccionalidad es de $83 \%$. En ningún caso la fuerza cortante máxima se presentó en las direcciones de las componentes de la excitación. Los momentos de volteo máximos se muestran en la Tabla 4. Al igual que para las fuerzas cortantes, considerar las dos componentes horizontales ortogonales en la base del tanque tiene efectos importantes e11el caso de movimientos como Viveros. No se reportan los valores de las respuestas anteriores para los registros de Central de Abastos debido a que los resultados de las ecuaciones lineales no son válidos. 
Tabla 3 Fuerzas cortantes máximas

\begin{tabular}{|c|c|c|c|c|}
\hline \multirow{3}{*}{ Movimiento } & \multicolumn{2}{|c|}{ Fuerza Cortante } & \multirow{3}{*}{$\begin{array}{l}\% \\
\text { Inc. }\end{array}$} & \multirow{3}{*}{$8^{\circ}$} \\
\hline & & & & \\
\hline & $\begin{array}{c}\text { Una excitación } \\
\text { horizontal }\end{array}$ & $\begin{array}{c}\text { Dos } \\
\text { excitaciones } \\
\text { ortogonales }\end{array}$ & & \\
\hline CU.NS & 5.0778 & \multirow[t]{2}{*}{6.5117} & 28 & \multirow[t]{2}{*}{86} \\
\hline CU.EW & 6.4976 & & 1 & \\
\hline VIV.NS & 7.2429 & \multirow[t]{2}{*}{10.5263} & 45 & \multirow[t]{2}{*}{317} \\
\hline VIV.EW & 7.2280 & & 46 & \\
\hline SCT.NS & 17.8883 & \multirow[t]{2}{*}{32.6490} & 83 & \multirow[t]{2}{*}{240} \\
\hline SCT.EW & 30.6394 & & 7 & \\
\hline TACY.NS & 6.2931 & \multirow[t]{2}{*}{7.2655} & 15 & \multirow[t]{2}{*}{203} \\
\hline TACY.EW & 5.4546 & & 33 & \\
\hline CALETA.NS & 24.7113 & \multirow{2}{*}{26.1263} & 6 & \multirow[t]{2}{*}{115} \\
\hline CALETA.EW & 254507 & & 3 & \\
\hline
\end{tabular}

\begin{tabular}{|c|c|c|c|c|}
\hline \multirow[b]{2}{*}{ Movimiento } & \multicolumn{2}{|c|}{ Momento de Volteo "t.m' } & \multirow[b]{2}{*}{$\begin{array}{l}\% \\
\text { Inc. }\end{array}$} & \multirow[b]{2}{*}{$8^{\circ}$} \\
\hline & $\begin{array}{c}\text { Una excitación } \\
\text { horizontal }\end{array}$ & $\begin{array}{c}\text { Dos } \\
\text { excitaciones } \\
\text { ortogonales }\end{array}$ & & \\
\hline CU.NS & 6.3035 & \multirow[t]{2}{*}{8.1248} & 29 & \multirow[t]{2}{*}{86} \\
\hline CU.EW & 8.1095 & & 1 & \\
\hline VIV.NS & 8.9020 & \multirow[t]{2}{*}{13.0591} & 46 & \multirow[t]{2}{*}{317} \\
\hline VIV.EW & 8.9550 & & 46 & \\
\hline SCT.NS & 22.3694 & \multirow[t]{2}{*}{40.8547} & 83 & \multirow[t]{2}{*}{243} \\
\hline SCT.EW & 38.3321 & & 7 & \\
\hline TACY.NS & 8.3315 & \multirow[t]{2}{*}{9.0547} & 9 & \multirow[t]{2}{*}{203} \\
\hline TACY.EW & 6.7233 & & 35 & \\
\hline CALETA.NS & 30.6850 & \multirow[t]{2}{*}{32.4560} & 6 & \multirow[t]{2}{*}{115} \\
\hline CALETA.EW & 31.6015 & & 3 & \\
\hline
\end{tabular}

Las Tablas 5, 6 y 7 muestran presiones hidrodinámicas en difere11tes puntos de la altu1·a del tanque para las componentes horizontales y vertical de los registros SCT, TACY y CALETA, respectivamente; las tablas listan también las presiones hidrodi11ámicas producto de la acción simultánéa de las tres componentes. En la base del tanque, las presiones debidas al componente vertical del registro SCT son del orden del 55\% y $85 \%$ de las que producen las componentes EW y NS, respectivamente. Par el movimiento TACY la contribución de la componente vertical de la aceleración en el cálculo de las presiones hidrodinámicas es despreciable. En el caso del registro de Caleta de Campos, las presiones debidas a la componente vertical en la base del tanque es del orden del $80 \%$ de la correspondiente a la componente $\mathrm{EW}$ y es un $25 \%$ mayor que la producida por la componente NS. Si comparamos las presiones en la base producidas por la acción de las tres componentes de excitación, encontramos que despreciar la contribución de la componente vertical resulta en errores del $25 \%$ y $30 \%$ en los casos de SCT y CALETA. 
Tabla 5 Presiones hidrodinámica s, registro sísmico SCT

\begin{tabular}{|c|c|c|c|c|}
\hline \multirow{2}{*}{$Z(\mathrm{~m})$} & \multicolumn{3}{|c|}{ Presiones Hidrodinámicas $/ \mathrm{t} / \mathrm{m}_{i}}$, \\
\cline { 2 - 3 } & SCT.NS & SCT.EW & \multicolumn{1}{|c|}{ SCT.VER } & NS+EW+VER \\
\hline 0.00 & 0.303 & 0.464 & 0.000 & 0.511 \\
\hline-0.25 & 0.305 & 0.465 & 0.145 & 0.537 \\
\hline-0.50 & 0.309 & 0.470 & 0.136 & 0.576 \\
\hline-0.75 & 0.317 & 0.478 & 0.221 & 0.615 \\
\hline-1.00 & 0.328 & 0.491 & 0.290 & 0.658 \\
\hline-1.25 & 0.344 & 0.508 & 0.355 & 0.709 \\
\hline-1.50 & 0.367 & 0.532 & 0.419 & 0.771 \\
\hline-1.75 & 0.399 & 0.563 & 0.490 & 0.846 \\
\hline-2.00 & 0.440 & 0.648 & 0.522 & 0.941 \\
\hline-2.25 & 0.499 & 0.774 & 0.532 & 1.063 \\
\hline-2.50 & 0.610 & 0.944 & 0.534 & 1.244 \\
\hline
\end{tabular}

Tabla 6 Presiones hidrodinámicas, registro sísmico TACY

\begin{tabular}{|c|c|c|c|c}
\hline \multirow{2}{*}{$\mathrm{Z}(\mathrm{m})$} & \multicolumn{3}{|c|}{ Presiones Hidrodinámicas $/$} & \\
\cline { 1 - 2 } 0.00 & TACYNS & TACY.EW & tlmL. & \\
\hline-0.25 & 0.186 & & TACY.VER & \\
\hline-0.50 & 0.186 & & NS+EW+VER & \\
\hline-0.75 & 0.188 & & 0.000 & \\
\hline-1.00 & 0.196 & 0.215 & & \\
\hline-1.25 & 0.202 & & 0.246 & \\
\hline-1.50 & 0.209 & 0.216 & 0.005 & \\
\hline-1.75 & 0.219 & & 0.247 & \\
\hline-2.00 & 0.231 & 0.218 & 0.010 & \\
\hline-2.25 & 0.245 & & & \\
\hline-2.50 & 0.263 & & 0.250 & \\
\hline & & 0.224 & 0.015 &
\end{tabular}

Tabla 7 Presiones hiddrodinámicas, regi\$®5 \$ísmico CALETA

\begin{tabular}{|c|c|c|c|c|}
\hline \multirow{3}{*}{$\overline{Z(m)}$} & & 0.231 & 0.020 & \\
\hline & \multicolumn{3}{|c|}{ Presiones Hidrodinámicas /tlm. } & \multirow{3}{*}{$\frac{\mathrm{NS}+\mathrm{EW}+\mathrm{VER}}{0.343}$} \\
\hline & CALETA.NS & \multirow{2}{*}{ CALETA $\frac{\text { LEW }}{0.334} .39 \mathrm{~W}$} & CALEFALVR & \\
\hline 0.00 & $0.2 \& 5$ & & 0.000 & \\
\hline-0.25 & 0.284 & 0.332 & 0.022 & 0.343 \\
\hline-0.50 & 0.280 & 0.30651 & 0.0249 & 0.340 \\
\hline-0.75 & 0.274 & 0.315 & 0.065 & 0.332 \\
\hline-1.00 & 0.265 & $0.303_{64}$ & 0.0 .77 & 0.328 \\
\hline-1.25 & 0.253 & $0.288^{\circ 4}$ & 0.109 & 0.326 \\
\hline-1.50 & 0.237 & 0.281 & 0.298 & 0.322 \\
\hline-1.75 & 0.218 & 0.277 .380 & 0.039 & 0.315 \\
\hline-2.00 & 0.194 & 0.262 & 0.174 & 0.317 \\
\hline-2.25 & 0.168 & $0.253 \mathrm{c}$ & 0.:-68 & 0.346 \\
\hline-2.50 & 0.164 & 0.272 & 0.218 & 0.388 \\
\hline & & 0.32182 & $\begin{array}{l}0.337 \\
0.049\end{array}$ & \\
\hline & & & 0.365 & \\
\hline
\end{tabular}


En la Figura 6 se n1uestra la variació11 de las presiones hidrostáticas, hidrodinán1icas e hidráulicas en la pared del tanque, para los movimientos sísmicos registrados en SCT, TACY y CALETA. La presión hidráulica es la resultante de las presiones hidrostática e hidrodinámica. En la pared del tanque, a la altura del nivel medio de la st1perficie del líquido, la presió11 hidrostática es nula y solamente existen presiones hidrodinámicas. A cierta altura por debajo del nivel medio del líquido las presiones 11idrostáticas e hidrodinámicas son iguales. Estos resultados indican que contrariamente a las recomenda ciones de algunos códigos, el diseño del espesor de los anillos superiores de tanques no se debe basar en el cálculo de esfuerzos debidos únicamente a la presión hidrostática. En la base del tanque la presión hidrostática es considerablemente mayor que la hidrodinámica, como se observa en el caso de los registros SCT y TACY. Sin embargo, en el caso del registro CALETA, la magnitud de la presión hidrodinámica en la base del tanque no se puede despreciar en comparación con la hidrostática; ésta es del orden de $75 \%$ de la presión hidraúlica. En consecuencia, la recon1endación de los códigos de diseña1 espesores con base en presiones hidrostáticas pod1.ía no ser apropiado incluso en la base del tanque.

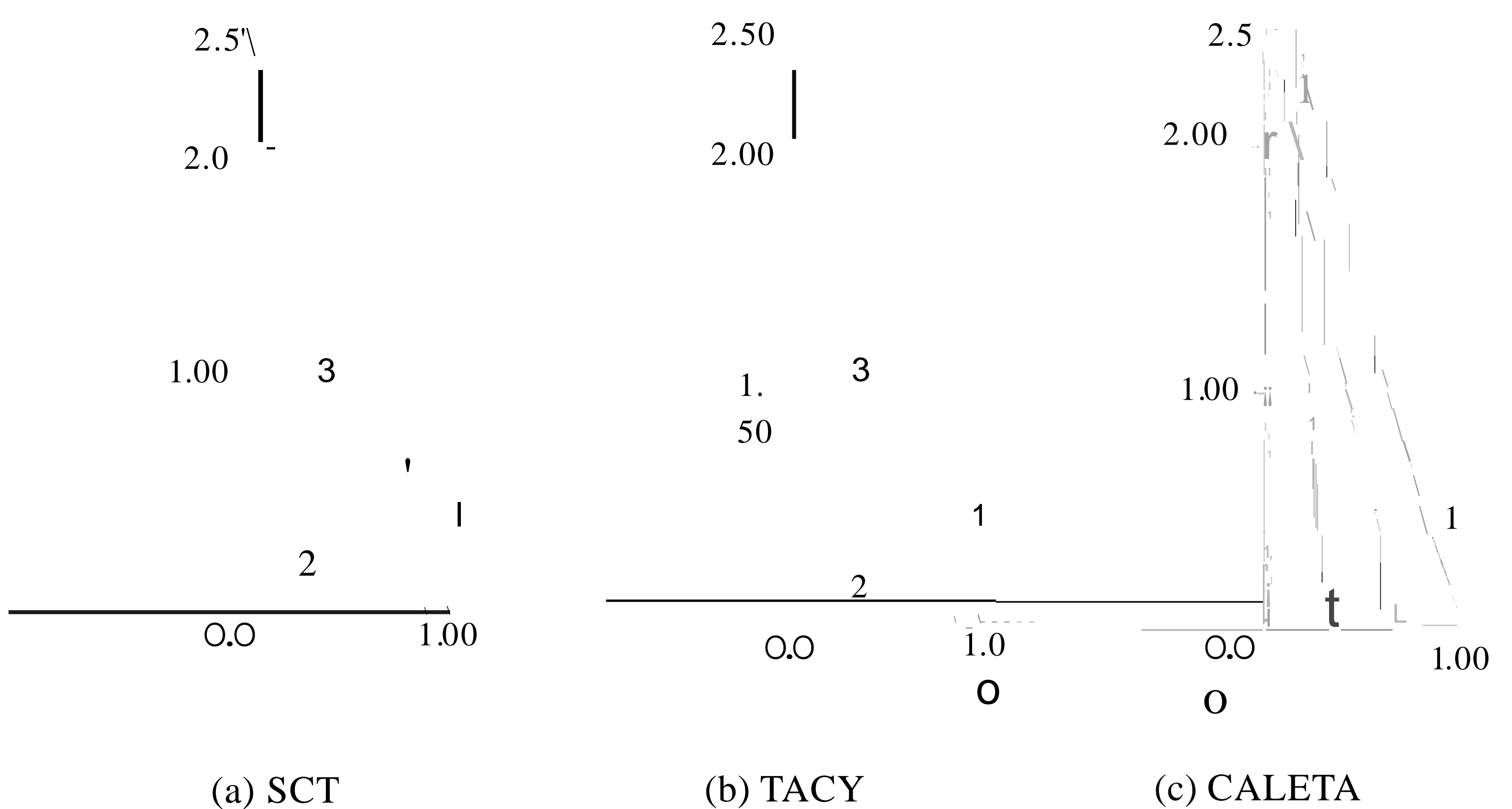

Fig 6. Distribución de presiones en la pared del tanque: (1) hidráulica, (2) hidrodinámica y (3) hidrostática

\section{ANÁLISIS EN RESONANCIA}

Con el fin de entender mejor el aparente comportamiento en resonancia del tanque del ejemplo de aplicación son1etido al movimiento registrado en Central de Abastos, se analizó el caso de una excitación de tipo armónica aplicada en su base ax(t)=iAeint donde $1=,-1, n$ es la frecuencia de la excitación y A su ahplitud. Se supone aquí que no se 
presentan efectos de interacción suelo-estructura, los cuales alargarían el periodo fundamental del sistema e incrementarían el amortiguamiento del sistema por la radiación de ondas al suelo. Estos efectos se traducirían en reducciones de las alturas de ola calculadas.

Considerando que en resonancia la contribución de la respuesta dinámica es en el modo funda1nental, la ecuación (31) se convierte en:

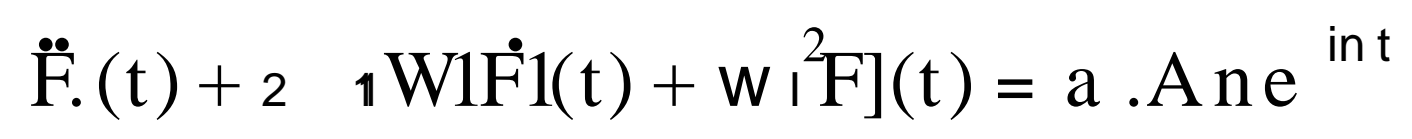

donde

$$
a_{1}=x_{1-1}^{2 a}=\frac{2}{1.8444^{2}-1} a=0.8332 a
$$

de manera que el sistema se puede modelar como un oscilador amortiguado de un grado de libertad tal como se indica en la Figura 7. La solución de la ecuación (52) para $\dot{\mathrm{F}}_{1}(\mathrm{t})$ es:

$$
\dot{\mathrm{F}}_{1}(\mathrm{t})=-\mathrm{Aa}_{1} \mathrm{G}(\mathrm{r}) \operatorname{sen}\left(\mathrm{Qt}+8_{0}\right)
$$

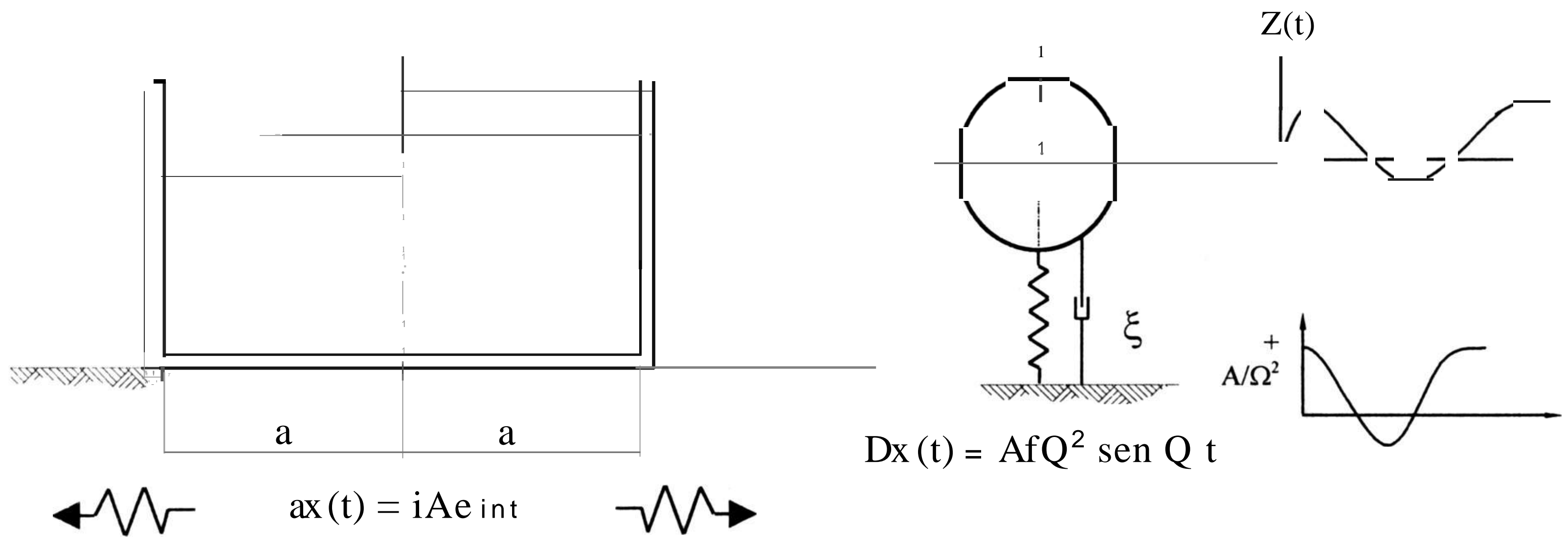

Fig 7. Tanque cilíndrico modelado como un sistema de I GDL sometido a una excitación armón $1 \mathrm{ca}$

En (54), G(r) es un factor de amplificación dinámico dado por

$$
G(r)=\frac{r^{2}}{\sqrt{\left(1-r^{2}\right)^{2}+\left(2 \xi_{1} r\right)^{2}}}
$$

y $8 \mathrm{n}$ es el ángulo de fase,

$$
e_{n}=\tan ^{-1}\left|\frac{21 r}{r^{2}-1}\right|
$$


donde $\quad \frac{\Omega}{\mathrm{W}_{\mathrm{i}}}$ es la relación entre la frecuencia de la excitación, $\Omega$ y la del oleaje en el modo fundamental, $\mathrm{w}_{1}$.

En $\mathrm{R}=\mathrm{a}$ y $8=0^{\circ}$ la altura de ola máxima se puede calcular como:

$$
8(\mathrm{a}, \mathrm{O}, \mathrm{t}) \text { máx }=-\frac{1}{\mathrm{~g}}-\mathrm{a} A \text { A sen } \ddot{\mathrm{u}} \mathrm{t}+\left.\dot{\mathrm{F}}_{1}(\mathrm{t})\right|_{\text {max }}
$$

Sustituyendo la solución de la ecuación (54) en (57) tenemos que

$$
8(\mathrm{a}, \mathrm{O}, \mathrm{t}) \text { máx }=\underset{\mathrm{g}}{\mathrm{A}}[\mathrm{f}(\mathrm{t}) \text { máx }]
$$

donde

$$
f(t)=[\operatorname{sen} \ddot{u} t+0.8332 G(r) \operatorname{sen}(\ddot{u} t+8 n)]
$$

Suponiendo que los movimientos sísmicos registrados en el Valle de México son armónicos, se calculó la altura de ola máxima para el tanque cilíndrico en estudio usando la ecuación (59). Se consideró un amortiguamiento crítico del líquido contenido en el tanque de $0.5 \%$. En la Tabla 8 se listan las características de los movimiento armónicos, la relación de frecuencias, el factor de amplificación y las alturas de ola máximas. Se puede observar que en el caso de los registros de la Central de Abastos el factor de amplificación dinámico es muy grande debido a la cercanía entre la frecuencia dominante de la excitación y la del modo fundamental del oleaje, verificándose el efecto de resonancia. Las alturas de ola máximas calculadas son iguales a 2.30 y $4.80 \mathrm{~m}$, y rebasan la altura del tanque. En consecuencia la teoría lineal es incapaz de predecir apropi adamente la respuesta del sistema en resonancia.

Tabla 8 Características de los movimientos armónicos y resultados

\begin{tabular}{|c|c|c|c|c|c|c|}
\hline Movimiento & Periodo (s) & Amáxlg & $\mathrm{n}(\mathrm{rad} / \mathrm{s})$ & $\mathrm{r}={ }_{\mathrm{w}}^{\mathrm{n}}$ & $\mathrm{G}(\mathrm{r})$ & 8 máx $(\mathrm{cm})$ \\
\hline CU.NS & $1.70-$ & 0.0281 & 3.6960 & 2.2817 & 1.2377 & 28.54 \\
\hline CU.EW & 0.95 & 0.0334 & 6.6139 & 4.0831 & 1.0638 & 31.49 \\
\hline VIV.NS & 0.58 & 0.0411 & 10.8330 & 6.6878 & 1.0228 & 38.00 \\
\hline VIV.EW & 0.54 & 0.0424 & 11.6355 & 7.1833 & 1.0197 & 39.16 \\
\hline SCT.NS & 2.0 & 0.0976 & 3.1416 & 1.9394 & 1.3621 & 104.18 \\
\hline SCT.EW & 2.0 & 0.169 & 3.1416 & 1.9394 & 1.3621 & 180.39 \\
\hline CA.NS & 3.6 & 0.0658 & 1.7453 & 1.0774 & 7.2031 & 230.09 \\
\hline CA.EW & 4.0 & 0.0688 & 1.5708 & 0.9697 & 15.5516 & 480.45 \\
\hline
\end{tabular}


La Figura 8 muestra la variación de la altura de ola máxima normalizada a óg, en la superficie libre del líquido en función de la relación de frecuencias. Cuando la frecuencia fundamental del oleaje en la superficie . libre del líquido es similar a la frecuencia de la excitación, como ocurre en el movimiento registrado en CA, el efecto de resonancia en la teoría lineal produce resultados que escapan al contexto físico del problema. Será necesario resolver el problema de oleaje no-lineal para predecir mejor las alturas de ola en resonancia; sin embargo, es previsible que en este caso las grandes alturas de ola en la superficie del líquido puedan producir daños importantes en las cubiertas.

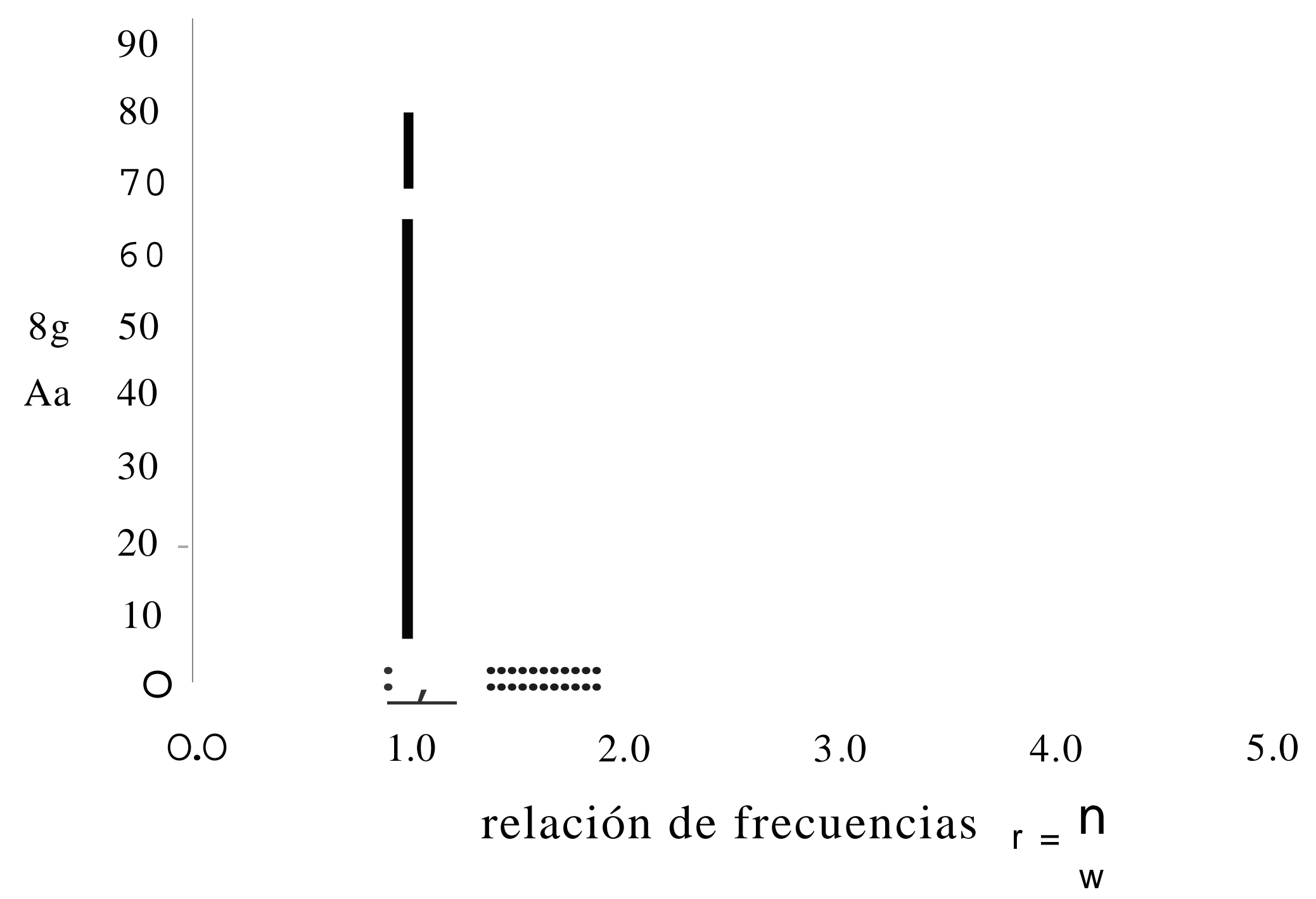

Fig 8. Amplitud de ola máxima normalizada vs relación de frecuencias

\section{CONCLUSIONES}

Se estudió la respuesta sísmica de tanques cilíndricos para almacenamiento de fluidos, de paredes rígidas y anclados a la cimentación. Se supuso que el fluido es ideal, irrotacional, incompresible y no viscoso. Las ecuaciones de movimiento se resolvieron en coordenadas cilíndricas y la respuesta se calculó paso a paso en el dominio del tiempo. La disipación de energía por amortiguamiento se modeló mediante un amortiguamiento de tipo viscoso. Se utilizaron registros del sismo del 19 de septiembre de 1985 en las estaciones Viveros, Ciudad Universitaria, Secretaría de Comunicaciones y Transporte, Central de Abasto, Tacubaya y Caleta de Campos.

Los resultados mostraron que la contribución de los modos superiores es importante para el cálculo de la altura de ola cuando el tanque es sometido a una excitación sísmica horizontal. Incrementos de respuesta por encima de un $20 \%$ se obtuvieron para algunos registros, y en particular para el registro SCT (componente NS) el incremento fue del orden del 84\%. Los efectos de bidireccionalidad de la excitación pueden contribuir significativamente en el cálculo de las fuerzas cortantes y momentos de volteo en la pared del tanque. En el caso del 
Respuesta sísmica de tanqtles cilíndricos para almacenamiento de líquidos

registro Viveros se obtuvo incrementos de respuesta por efectos de bidireccionali dad del orden del $45 \%$. La componente vertical de la excitación sísmica puede contribuir al aumento de las presiolles hidrodinán1icas en la base del tanque. El error por despreciar dicha contribución puede ser del orde11del $30 \%$.

Se compararon las presiones hidráulicas e hidrostática; en la porción superior de la pared del tanque la presión hidráulica es mayor que la hidrostática. En el caso de movimientos como el registrado en Caleta, la presión hidráulica puede no ser despreciable comparada con la hidrostática en la base de la pared tanque. En consecuencia, la recomendación de algunos códigos de diseño de calcular espesores considerando solamente la presión hidrostática no es apropiada no sólo en los anillos superiores, sino también en la base del tanque.

Se encontró que para el registro de Central de Abastos, en el que la frecuencia don1inante de la excitación es cel-cana a la frecuencia del modo fundamental del oleaje en la superficie libre, se produjo el fenómeno de resonancia. La teoría lineal no es capaz de predecir apropiad amente la respuesta en este caso y resulta en alturas de ola que violan las condiciones físicas del problema. En el caso de resonancia, es necesario considerar los efectos hidrodinámicos no lineales pára estudiar la respuesta sísmica de este tipo de estructuras.

\section{AGRADECIMIENTO}

Loa autores agradecen el apoyo del Instituto Mexicano del Petróleo para el desarrollo de este trabajo de investigación así como el patrocinio de la beca doctoral del primer autor. El segundo autor agradece también a la Dirección General de Asuntos del Personal Académico de la UNAM el apoyo a los proyectos de investigación patrocinados en el programa PAPIIT. Se extiende un reconocimiento a los revisores anónimos por sus valiosos comentarios y sugerencias.

\section{REFERENCIAS}

1. Case K. M. Parkinson W. C. (1957)."Damping of surface waves in an incompressible liquid", Joumal of Fluid Mechanics, Vol. 10, N 1,172-184.

2. Chen W., Haroun M. y F. Liu ( 1996). "Large amplitude liquid sloshing in seismically excited tanks", Earthqauke Engineering and Structural Dynamics, Vol. 25, 653-669.

3. Faltinsen O. M. (1978)."A numerical nonlinear method of sloshing in tanks with two dimensional flow", Joumal of Ship Research, Vol. 22, No 3, 193-202.

4. Gaunquing Ch. (1984). "Why the elephant's foot phenomenon of liquid storage tanks happened", Proceedings Eighth World Conference on Earthquake Engineering, San Francisco, CA, Vol. 7, 445-452.

5. Haroun M. A. (1983). "Vibration studies and tests of liquid storage tanks", Earthquake Engineering and Structural Dynamics, Vol. 11, 179-206.

6. Housner G. W. (1957). "Dynamic Pressures on Accelerated fltlid containers", Bulletin of the Seismological Society of America, Vol . 47, 15-35. 
7. Housner G. W. y Haroun M. A. (1980). "Dynamic analyses of liquid storage tanks", Turkish National Committee on Earthquake Engineering, Proceedings Seventh World Conference on Earthquake Engineering, Istanbul, Turkey, Vol. 8, 431-438.

8. Jennings P. C. (1971)."Damage of storage tanks, engineering features of the San Femado

Earthquake, Feb. 9, 1971", Earthquake Engineering Research Laboratory, Cal. Tech.

9. Keulegan G. H. (1958). "Energy dissipation in standing waves in rectangular basin", Joumal of Fluid Mechanics, Vol. 9, 153-158.

10. Le Méhauté B. (1976). "An introduction to hydrodynamic and water waves", SpringerVerlag.

11. Manos G. C. y Clough R. W. (1985). "Tank damage during the May 1983 Coalinga Earthquake", Earthquake Engineering and Structural Dynamics, Vol. 13, 449-466.

12. Mei C. C. y Liu L. F. (1973). "The damping of surface gravity waves in a bounded liquid", part 2, Joumal of Fluid Mechanics, Vol. 59, 239-256.

13. Niwa A. y Cluogh R. W. (1982). "Buckling of cylindrical liquid-storage tanks under earthquake loading", Earthquake .Engineering and Structural Dynamics, Vol. 1O, 107-122.

14. Priestley M. J. N. (Editor) (1986). "Seismic Design of Storage Tanks", New Zealand National Society of Earthquake Engineering (NZNSEE).

15. Rogge T. R. y Weiss H. J. (1966). "An approximate nonlinear analysis of the stability of sloshing modes under translational and rotationalexcitation", NASA CR-220, San Diego.

16. Sakar F. N. y Ogawa H. (1984). "Sloshing behavior of floating-roof oil storage tanks", Computer and Structures Vol. 19, N 1-2, 183-192

17. Veletsos A. S. y Shivakumar P. (1993). "Sloshing response of layered liquids in rigid tanks", Earthquake Engineering and Structural Dynamics, Vol. 22, 801-821.

18. Veletsos A. S. y Shivakumar P. (1997). "Dynamic response of tanks containing liquids or solids", Rice University, Houston, Texas, USA.

19. Veletsos A. S. y Yang J. Y. (1977). "Earthquake response of liquid storage tanks", Advances in Civil Engineering through Engineering Mechanics, Proceedings Second Annual Engineering Mechanics Division Specialty Conference, ASCE, 124.

20. Veletsos A. S. y Kumar A. (1984). "Dynamic of vertically excited liquid storage tanks", Proceeding of the VII WCEE, San Francisco, CA, USA.

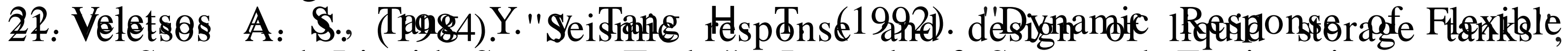

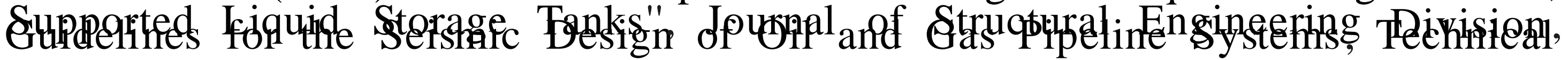
ASGFEil1br, 26ftetime Earthquake Engineering, ASCE, New York, 255-370 y 443461. 